\title{
The motion of a deformable drop in a second-order fluid
}

\author{
By P. C.-H. CHAN AND L. G. LEAL \\ Department of Chemical Engineering, California Institute of Technology, Pasadena, 91125
}

(Received 10 April 1978 and in revised form 20 September 1978)

The cross-stream migration of a deformable drop in a unidirectional shear flow of a second-order fluid is considered. Expressions for the particle velocity due to the separate effects of deformation and viscoelastic rheology are obtained. The direction and magnitude of migration are calculated for the particular cases of Poiseuille flow and simple shear flow and compared with experimental data.

\section{Introduction}

Recently, the dynamics of fluid-like materials which consist of two distinct phases has been the subject of intense investigation from both an experimental and a theoretical point of view. The present paper is concerned with one aspect of this general problem; namely, the cross-flow migration of small particles in a suspension which is undergoing a shearing flow at small Reynolds numbers. When cross-flow migration occurs, the particle concentration distribution becomes non-uniform with important consequences in the overall flow characteristics of the suspension. In addition, any effective material property whose magnitude is dependent upon the local particle concentration will also become non-uniform. One technological problem where such effects are important is the processing of two-phase (or fibre/particle filled) plastics. In this case, the particulate phase is ordinarily added to the polymer matrix in order to change one or more of the bulk properties of the composite material. However, in some cases, the particulate is simply added as a filler in order to decrease the quantity of polymer which is required per unit volume of product; for this purpose, the least expensive filler is, of course, small air bubbles. For these composite-media processing problems, the objective is usually a uniform concentration of particulate in the final product; however, in the case of added 'filler' material, one might alternatively require that the concentration of particulate at the surfaces of the finished product be small (or zero), in order to enhance its appearance. Regardless of the detailed objectives in processing applications, however, it is clearly important at the design and development stage to understand the mechanisms and dynamies of cross-flow particulate migration for rigid particles, bubbles or drops in a non-Newtonian suspending fluid. The present investigation is thus concerned with cross-flow migration for neutrally buoyant drops which are suspended in a non-Newtonian fluid that is undergoing a quadratic, unidirectional shearing flow. In this case, in the absence of external body forces, the mechanism for any particle migration must be purely hydrodynamic in origin. The focus of our present work is the development of further understanding of these hydrodynamically-induced migration mechanisms. 
Prior to outlining the research which is reported here, we will briefly review those previous experimental and theoretical studies which pertain directly to the problem of cross-stream migration of drops in either Newtonian or non-Newtonian fluids. The migration of drops of one Newtonian fluid in a second Newtonian fluid at zero (i.e. very small) Reynolds number was studied experimentally by Goldsmith \& Mason (1962) for three-dimensional Poiseuille flows, and by Karnis \& Mason (1967) for Couette flows. In both cases, migration due to drop deformation was observed to occur toward the centre-line, whereas no migration will occur if rigid spheres are used in the experiments. In an attempt to explain this phenomenon, Chaffey, Brenner \& Mason $(1965,1967)$ considered the motion of a deformable drop in a simple shear flow near a single wall using the method of reflexions. They found that the drop would migrate away from the wall, in apparent qualitative agreement with the experimental observations. Later. Haber \& Hetsroni (1971), Wohl \& Rubinow (1974) and Wohl (1976) all considered the motion of a deformable drop in a unidirectional shear flow with a shear gradient, with wall effects being neglected except in the determination of the bulk velocity profile. With the exception of Haber \& Hetsroni (1971) who apparently made algebraic errors, these theories also showed migration in the direction of the centre-line in Poiseuille flow, though obviously the detailed dependence on system parameters is different from the linear shear flow result of Chaffey et al. $(1965,1967)$. It may be noted that neither theory provides a good fit to the actual experimental trajectory data - in spite of statements to the contrary in the original papers. In addition, the results of Wohl (1976) and Wohl \& Rubinow (1974) show a predicted migration velocity for three-dimensional Poiseuille flow which is approximately ten times as large as the predicted value in two-dimensional Poiseuille flow, all other conditions being exactly the same. In our opinion, this result is contrary to one's intuitive sense, and casts considerable doubt on the accuracy of both analyses. Finally, it may be reiterated that the Chaffey et al. $(1965,1967)$ theory is for a single plane boundary, whereas the available experimental work pertains primarily to circumstances where there are either two walls or a circular tube as the bounding surface. On the experimental side, it should also be remarked that the range of parameters tested so far is not extensive; for example, the ratio of internal to external fluid viscosities was always close to zero in the experiments of Mason and co-workers.

Unlike the case of two Newtonian fluids where migration occurs (at zero Reynolds number) only as a result of particle shape deformation, cross-flow migration is known to occur in non-Newtonian fluids for both rigid and deformable particles. Experimental studies of neutrally buoyant spheres and Newtonian drops in viscoelastic, as well as purely viscous, fluids have been reported by Gauthier, Goldsmith \& Mason $(1971 a, b)$, following an earlier study by Karnis \& Mason (1966). For a rigid sphere in a viscoelastic fluid, migration is observed to occur in the direction of decreasing absolute shear rate for both Couette and Poiseuille flows. Newtonian drops, on the other hand, migrate toward the centre-line in Poiseuille flow but at a rate greater than that for either a rigid particle in a viscoelastic fluid or a drop in a Newtonian fluid, while in a Couette flow, they migrate to an intermediate position between the 'centre-line' and the outer cylinder wall. These results were interpreted qualitatively by Mason and coworkers as resulting from a superposition of the viscoelastic migration effect for a particle of spherical shape and the deformation induced migration of a Newtonian drop in a Newtonian fluid. Unlike the Newtonian migration of a deformed drop, there 
have been relatively few theoretical investigations which pertain to cross-flow migration in a non-Newtonian fluid. This is most likely a result of anticipated uncertainties in the selection of a reasonable constitutive model for non-viscometric flows, as well as the obvious difficulty in solving the equations of motion after the choice has been made. In our opinion, however, the usual constitutive equation dilemma is frequently not as serious for low Reynolds number motions involving small particles, as for nonviscometric flows in general. This is due to the fact that the creeping motion solutions for a Newtonian fluid in these cases often exhibit an 'indeterminacy' in the configuration of the particle relative either to the undisturbed bulk flow or to the container boundaries, and it is the 'resolution' of this 'indeterminacy' which is often the primary goal of the non-Newtonian analysis. For this type of problem it is sufficient, in at least a qualitative sense, to consider the influence of small instantaneous departures from Newtonian fluid behaviour acting over a large time (i.e. to consider particle motion for a long period of time in a nearly Newtonian fluid). Two important examples of 'configurational indeterminacies' which may be treated with an analysis of this type are the steady-state orbit of rotation of an axisymmetric particle in simple shear flow (Leal 1975) and the steady-state position of a particle relative to container boundaries in a unidirectional shearing flow. The latter is the problem which we are investigating in the present paper. It is essential to note that the appropriate constitutive model for non-viscometric flows which are nearly Newtonian is, unlike the case of a general non-viscometric flow, well-known to be the Rivlin-Ericksen fluid provided the motion is both weak and slow in a rheological sense. This model may be obtained, via the so-called 'retarded motion' expansion, from almost all of the currently popular nonlinear constitutive models. The case of the lateral migration of a rigid sphere in a quadratic, unidirectional undisturbed flow was considered previously by Ho \& Leal (1976), who used the second-order Rivlin-Ericksen fluid model, thereby including normal stress contributions to the particle motion, but excluding shear-rate dependent viscosity. The analysis of Ho \& Leal (1976) was considerably simplified, not only by use of the second-order fluid constitutive model, but also by employing the reciprocal theorem approach of Cox \& Brenner (1968) and Ho \& Leal (1974) to enable the migration velocity to be calculated without any need to determine the non-Newtonian contribution to the velocity and pressure fields in the suspending fluid. The result of Ho \& Leal's (1976) theory predicts migration toward the outer cylinder and is therefore in apparent qualitative agreement with available experimental data.

In the present paper, we consider the related problem of the migration of a fluid drop in a unidirectional shearing flow, both with and without shear-rate gradients. Both the suspending fluid and the fluid inside the drop are assumed to be adequately modelled as second-order fluids - thus extending the domain of application somewhat beyond even the available experiments where the drop fluid was always Newtonian. The primary thrust of our present research is a systematic assessment of the coexisting roles of drop deformation and viscoelastic fluid behaviour in the migration of a drop. In effect, we investigate the relevance of Gauthier et al.'s (1971 $a, b)$ 'explanation' of the existing experimental observations which suggest an 'additive' effect of deformation in a Newtonian fluid and viscoelastic behaviour for a spherical drop. In so doing, we re-examine the problem of deformation-induced migration in a Newtonian fluid, as well as the more general non-Newtonian problem described above. 
In the following two sections, we shall outline the formulation of the problem, and obtain, by a perturbation expansion and the reciprocal theorem, a general expression for the migration velocity. In $\$ 4$, we obtain solutions for the Newtonian velocity fields that are required for evaluation of the migration velocity formula. This includes the motion of a deformable drop in a unidirectional shearing flow which is bounded by two plane walls, and the translation of a spherical drop through a quiescent fluid normal to two parallel plane walls. The migration velocity is evaluated and discussed in various limiting circumstances in $\$ \S 5-7$. In the case of migration in a quadratic velocity profile, we also use the method of Chan \& Leal (1977) to generalize our results to a general (three-dimensional) quadratic shear flow. Finally, in §8, we compare our results with the experimental observations of Mason and his co-workers.

\section{Formulation of the problem}

We consider the motion of a neutrally buoyant drop freely suspended in a fluid which is undergoing a unidirectional, quadratic shearing flow. The fluid is confined between two parallel plane walls separated by a distance $d$; hence the undisturbed flow is assumed to be two-dimensional. The two fluids are both assumed to be RivlinEricksen fluids, as discussed above, with zero shear viscosity $\mu_{0}$ for the suspending phase and $\tilde{\mu}_{0}$ for the fluid inside the drop. The whole motion is further assumed to be dominated by viscous and pressure effects, so that the inertial terms in the equations of motion can be neglected entirely. In order to write the governing differential equations and boundary conditions in non-dimensional form, we define a characteristic length $a$ and a characteristic velocity $G a$, where $a$ is the radius of the drop at zero deformation and $G$ is an average shear rate for the bulk flow. The stress tensors for the two fluids are non-dimensionalized using $\mu_{0} G$ and $\tilde{\mu}_{0} G$ respectively. In addition, we choose a co-ordinate reference system with origin $O$ which is fixed, for convenience, at the centroid of the drop, thus translating relative to a fixed reference frame with the velocity of the drop, which we shall denote by $\hat{\mathbf{U}}_{s}$. The position vector at material point $R$, measured relative to $O$, will be denoted as $\mathbf{x}$, while the complete dimensionless velocity, pressure and stress distributions, including the disturbance motion induced by the particle, are denoted in the two fluids as $(\mathbf{U}, P, \mathbf{S})$ and $(\widetilde{\mathbf{U}}, \widetilde{P}, \widetilde{\mathbf{S}})$ respectively.

With these conventions and assumptions, the equations of motion for the suspending fluid may be written in the familiar form

$$
\nabla . \mathbf{S}=0, \quad \nabla . \mathbf{U}=\mathbf{0},
$$

where $\quad \mathbf{S}=-P \mathbf{I}+\mathbf{D}_{(1)}+\lambda\left[\mathbf{D}_{(1)} . \mathbf{D}_{(1)}+\epsilon_{1} \mathbf{D}_{(2)}\right]+\lambda^{2}\left[\epsilon_{2}\left(\mathbf{D}_{(1)}: \mathbf{D}_{(1)}\right) \mathbf{D}_{(1)}\right.$

$$
\left.+\epsilon_{3} \mathbf{D}_{(3)}+\epsilon_{4}\left(\mathbf{D}_{(1)} \cdot \mathbf{D}_{(2)}+\mathbf{D}_{(2)} \cdot \mathbf{D}_{(1)}\right)\right]+O\left(\lambda^{3}\right)
$$

and $\mathbf{D}_{(n)}$ are Rivlin-Ericksen tensors given by

$$
\left.\begin{array}{l}
\mathbf{D}_{(1)}=\nabla \mathbf{U}+(\nabla \mathbf{U})^{T}, \\
\mathbf{D}_{(2)}=\frac{\partial}{\partial t} \mathbf{D}_{(\mathbf{1})}+\mathbf{U} \cdot \nabla \mathbf{D}_{(\mathbf{1})}+\mathbf{D}_{(1)} \cdot(\nabla \mathbf{U})^{T}+\nabla \mathbf{U} \cdot \mathbf{D}_{(\mathrm{1})}, \\
\mathbf{D}_{(3)}=\frac{\partial}{\partial t} \mathbf{D}_{(2)}+\mathbf{U} \cdot \nabla \mathbf{D}_{(2)}+\mathbf{D}_{(2)} \cdot(\nabla \mathbf{U})^{T}+\nabla \mathbf{U} \cdot \mathbf{D}_{(2)} \cdot
\end{array}\right\}
$$


Here $\lambda$ and $\epsilon_{1}$ are dimensionless parameters, defined as $\lambda=\phi_{3} G / \mu_{0}$ and $\epsilon_{1}=\bar{\phi}_{2} / \phi_{3}$ respectively $\left(\phi_{2}\right.$ and $\phi_{3}$ are dimensional normal stress coefficients; cf. Ho \& Leal 1976). We note that $\lambda$ is effectively the ratio of an intrinsic relaxation time scale for the fluid to the convective time scale of the fluid's motion. As indicated in (2.2) it will be assumed that $\lambda$ is small in the present analysis so that the second-order fluid model is applicable and non-Newtonian contributions to the fluid's behaviour are automatically assumed to be small. $\epsilon_{1}$, on the other hand, is of order unity (see $\$ 5.1$ ).

Similarly for the fluid inside the drop, we obtain

$$
\nabla . \tilde{\mathbf{S}}=0, \quad \nabla . \tilde{\mathbf{U}}=0,
$$

where

$$
\begin{aligned}
\widetilde{\mathbf{S}}= & -\tilde{P} \mathbf{I}+\widetilde{\mathbf{D}}_{(\mathbf{1})}+\tilde{\lambda}\left[\widetilde{\mathbf{D}}_{(1)} \cdot \widetilde{\mathbf{D}}_{(1)}+\tilde{\epsilon}_{1} \widetilde{\mathbf{D}}_{(2)}\right]+\tilde{\lambda}^{2}\left[\tilde{\epsilon}_{2}\left(\widetilde{\mathbf{D}}_{(1)}: \widetilde{\mathbf{D}}_{(1)}\right) \widetilde{\mathbf{D}}_{(1)}+\tilde{\epsilon}_{3} \widetilde{\mathbf{D}}_{(3)}\right. \\
& \left.+\tilde{\epsilon}_{4}\left(\tilde{\mathbf{D}}_{(1)} \cdot \widetilde{\mathbf{D}}_{(2)}+\widetilde{\mathbf{D}}_{(2)} \cdot \widetilde{\mathbf{D}}_{(1)}\right)\right]+O\left(\tilde{\lambda}^{3}\right)
\end{aligned}
$$

with $\widetilde{\mathbf{D}}_{(n)}$ defined analogously to $\mathbf{D}_{(n)}$, but using $\tilde{\mathbf{U}}$ instead of $\mathbf{U}$. In this case, $\tilde{\lambda}$ and $\tilde{\epsilon}_{\mathbf{1}}$ are defined using quantities relevant to the drop fluid. In this paper, we are interested in studying the case where the non-Newtonian properties of both fluids contribute to migration. The exact relationship between $\lambda$ and $\tilde{\lambda}$ in this situation will be considered in $\S 5$; for now, we shall simply assume that they are of the same order of magnitude.

We next turn to the undisturbed bulk flow. It is obvious that the undisturbed velocity, pressure and stress fields, $(\mathbf{V}, Q, \mathbf{T})$, together with the corresponding RivlinEricksen tensors $\mathbf{E}_{(n)}$, should also be assumed to satisfy creeping flow equations analogous to (2.1)-(2.3). These equations will not be repeated here. As indicated above, we assume that the bulk flow, when measured relative to fixed laboratory coordinates, is steady, unidirectional and two-dimensional. Since we are interested in shearing flows with a shear gradient, we may write the undisturbed flow relative to reference point $O$ as

with

$$
\mathbf{V}=\left(\alpha+\beta x_{3}+\gamma x_{3}^{2}\right) \mathbf{e}_{1}-\hat{\mathbf{U}}_{s}
$$

$$
Q=2 \gamma x_{1}+\left(\beta+2 \gamma x_{3}\right)^{2}\left(1+2 \epsilon_{1}\right) \lambda+12 \gamma x_{1}\left(\beta+2 \gamma x_{3}\right)^{2}\left(\epsilon_{2}+\epsilon_{4}\right) \lambda^{2}+\text { constant }
$$

Examples of flow types described by (2.6a) and (2.6b) include the simple, linear shear flow and the plane Poiseuille flow, both of which are illustrated in figure 1. For the simple shear flow, the coefficients are

$$
\alpha=V_{w} s, \quad \beta=V_{w} \zeta, \quad \gamma=0,
$$

whereas for the Poiseuille flow, they are

$$
\alpha=4 V_{\max } s(1-s), \quad \beta=4 V_{\max }(1-2 s) \zeta, \quad \gamma=-4 V_{\max } \zeta^{2} .
$$

$V_{w}$ and $V_{\max }$ are both measured relative to the fixed laboratory reference frame. In (2.7) and (2.8), $s$ is the dimensionless distance from a wall and $\zeta$ is the drop radius to gap width ratio, given by $\zeta=a / d$. Although the assumption of a two-dimensional undisturbed flow may seem unduly restrictive, we have previously shown (Chan \& Leal 1977) that the results may be extended to the corresponding three-dimensional flows, provided that hydrodynamic interactions between the particle and bounding wall are negligible. In that paper, a general method was developed by which the motion of a spherical particle in a general quadratic unidirectional flow of a second-order fluid was obtained completely from the detailed results of Ho \& Leal (1976) for the 

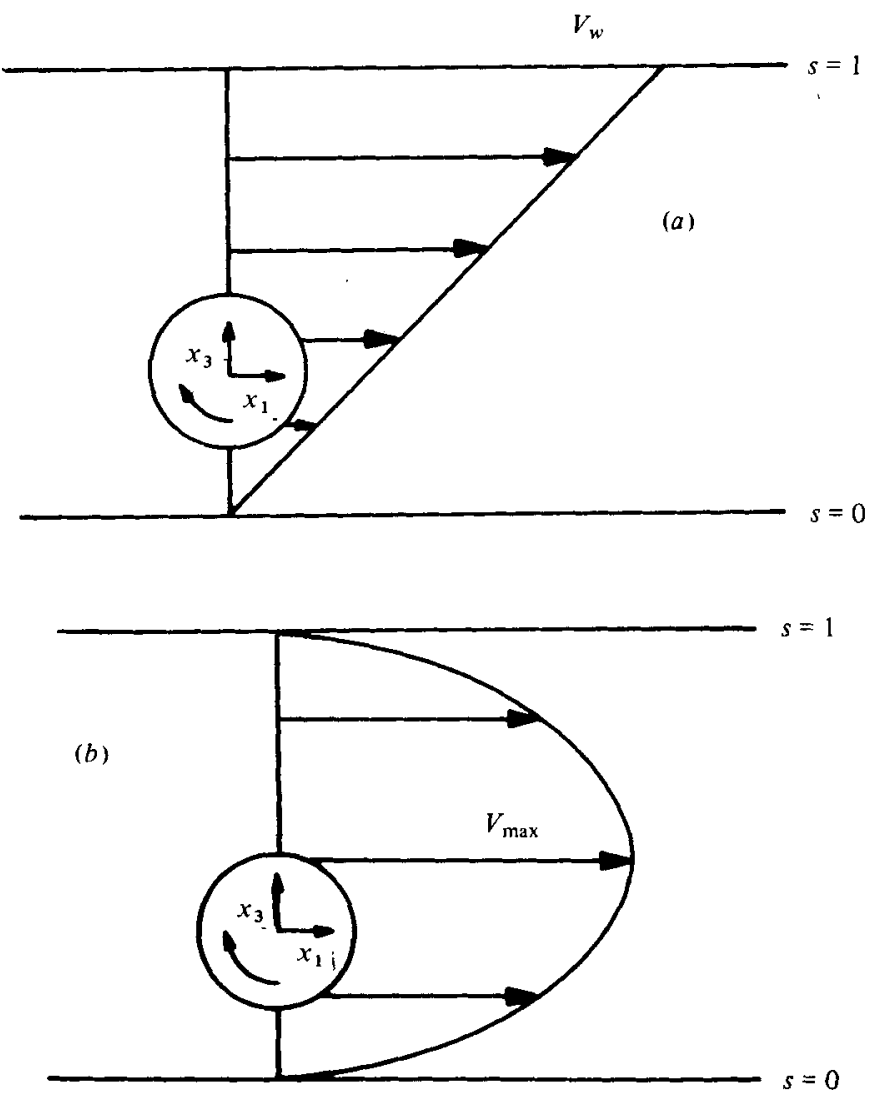

Figure 1. A side view of a drop in: (a) a simple shear flow; (b) a two-dimensional Poiseuille flow.

particle motion in a two-dimensional quadratic, unidirectional flow. In the present work, the method of Chan \& Leal (1977) is again applied to extend our detailed results for a two-dimensional quadratic unidirectional undisturbed flow to the cases of a three-dimensional Poiseuille flow and of a Couette flow. The latter are of particular interest because most of the experimental studies of particle migration have been carried out for Poiseuille and Couette flows. The initial choice of a two-dimensional undisturbed flow geometry is made largely due to the relative simplicity of the resulting analysis.

For our present case, the boundary conditions at large distances from the drop are

$$
\left.\begin{array}{ll}
\mathbf{U} \rightarrow \mathbf{V} & \text { as } r=|\mathbf{x}| \rightarrow \infty, \\
\mathbf{U}=V_{w} \mathbf{e}_{1}-\hat{\mathbf{U}}_{s} & \text { on the walls. }
\end{array}\right\}
$$

On the surface of the deformed drop

$$
\begin{aligned}
\mathbf{U} & =\widetilde{\mathbf{U}} \\
\mathbf{U} \cdot \mathbf{n} & =\widetilde{\mathbf{U}} \cdot \mathbf{n}=0 \\
\mathbf{S} \cdot \mathbf{n} & =\kappa \tilde{\mathbf{S}} \cdot \mathbf{n}+\frac{1}{\delta}\left(\frac{1}{R_{1}}+\frac{1}{R_{2}}\right) \mathbf{n} .
\end{aligned}
$$


Here $\kappa$ is the viscosity ratio (i.e. $\kappa=\tilde{\mu}_{0} / \mu_{0}$ ) whereas $R_{1}$ and $R_{2}$ are the principal radii of curvature. $\delta$ is a comparison between viscous forces and the interfacial tension $\sigma$, and is given by $\delta=a \mu_{0} G / \sigma$. In the present work, we shall restrict our attention to the case of small deformations from the spherical shape, with the spherical shape being preserved by interfacial tension. Hence, we shall assume that $\delta$ is a small parameter. Thus, in the perturbation expansion which follows, we shall adopt the procedures outlined by Taylor $(1932,1934)$ and Frankel \& Acrivos (1970), in which the velocity, kinematic and shear stress conditions are satisfied at each order, and the deformation of the drop is then calculated using the normal stress condition, $(2.10 \mathrm{c})$.

It should be noted that a second asymptotic limit exists where the drop shape remains near to spherical; namely, the case of a very viscous drop (i.e. $\kappa \rightarrow \infty$ ) which was also considered by Taylor (1934) and Frankel \& Acrivos (1970). There, the velocity and stress continuity conditions are satisfied on the surface of the drop at each order, and the non-zero normal velocity which results must be balanced by a deformation term. It can be easily shown in this case that the migration velocity to $O(1 / \kappa)$ is identically zero, due to fore-aft symmetry of the deformed drop plus alignment of its major axis with the axis of the undisturbed velocity field. Thus no further consideration will be given to deformation-induced migration in the limit $\kappa \rightarrow \infty$ in the present paper. The migration due to deformation in the limit $\delta \ll 1$ which we shall study is restricted to $\kappa<O(1 / \delta)$. On the other hand, the Newtonian velocity fields in $\S 4$, and the normal stress-induced migration calculations for a spherical drop in $\$ 5$ are both valid for all values of $\kappa$.

We now proceed formally to the solution of our problem, via a double asymptotic expansion in $\lambda$ and $\delta$. Thus, we let

$$
1 \gg \lambda, \delta \gg \lambda^{2}, \lambda \delta, \delta^{2} \ldots
$$

and write, for the particle velocity

$$
\hat{\mathbf{U}}_{s}=\hat{\mathbf{U}}_{s}^{(\theta)}+\lambda \hat{\mathbf{U}}_{s}^{(\lambda)}+\delta \hat{\mathbf{U}}_{s}^{(\delta)}+\lambda^{2} \hat{\mathbf{U}}_{s}^{(\lambda \lambda)}+\lambda \delta \hat{\mathbf{U}}_{s}^{(\lambda \delta)}+\delta^{2} \hat{\mathbf{U}}_{s}^{(\delta \delta)}+\ldots
$$

$\hat{\mathbf{U}}_{s}^{(0)}$ is the translational velocity of a Newtonian, spherical drop in a two-dimensional, quadratic shear flow of a Newtonian fluid, whereas $\hat{U}_{s}^{(\lambda)}$ represents the non-Newtonian (normal-stress) contribution to the translation of a spherical drop, and so on. We may also write down formal expansions for the velocity, pressure and stress fields. For the suspending phase, these are

$$
\left.\begin{array}{l}
\mathbf{U}=\mathbf{U}^{(0)}+\lambda \mathbf{U}^{(\lambda)}+\delta \mathbf{U}^{(\delta)}+\lambda^{2} \mathbf{U}^{(\lambda \lambda)}+\lambda \delta \mathbf{U}^{(\lambda \delta)}+\delta^{2} \mathbf{U}^{(\delta \delta)}+\ldots, \\
P=P^{(0)}+\lambda P^{(\lambda)}+\delta P^{(\delta)}+\lambda^{2} P^{(\lambda \lambda)}+\lambda \delta P^{(\lambda \delta)}+\delta^{2} P^{(\delta \delta)}+\ldots, \\
\mathbf{S}=\mathbf{S}^{(0)}+\lambda \mathbf{S}^{(\lambda)}+\delta \mathbf{S}^{(\delta)}+\lambda^{2} \mathbf{S}^{(\lambda \lambda)}+\lambda \delta \mathbf{S}^{(\lambda \delta)}+\delta^{2} \mathbf{S}^{(\delta \delta)}+\ldots,
\end{array}\right\}
$$

whereas for the fluid inside the drop, we obtain

$$
\left.\begin{array}{l}
\tilde{\mathbf{U}}=\tilde{\mathbf{U}}^{(0)}+\lambda \tilde{\mathbf{U}}^{(\lambda)}+\delta \tilde{\mathbf{U}}^{(\delta)}+\lambda^{2} \widetilde{\mathbf{U}}^{(\lambda \lambda)}+\lambda \delta \tilde{\mathbf{U}}^{(\lambda \delta)}+\delta^{2} \tilde{\mathbf{U}}^{(\delta \delta)}+\ldots, \\
\tilde{P}=\frac{1}{\delta} \tilde{P}^{(1 / \delta)}+\tilde{P}^{(0)}+\lambda \tilde{P}^{(\lambda)}+\delta \tilde{P}^{(\delta)}+\lambda^{2} \tilde{P}^{(\lambda \lambda)}+\lambda \delta \tilde{P}^{(\lambda \delta)}+\delta^{2} \tilde{P}^{(\delta \delta)}+\ldots, \\
\tilde{\mathbf{S}}=\frac{1}{\delta} \tilde{\mathbf{S}}^{(1 / \delta)}+\tilde{\mathbf{S}}^{(0)}+\lambda \tilde{\mathbf{S}}^{(\lambda)}+\delta \tilde{\mathbf{S}}^{(\delta)}+\lambda^{2} \tilde{\mathbf{S}}^{(\lambda \lambda)}+\lambda \delta \tilde{\mathbf{S}}^{(\lambda \delta)}+\delta^{2} \tilde{\mathbf{S}}^{(\delta \delta)}+\ldots
\end{array}\right\}
$$


Here, $\tilde{P}^{(1 / \delta)}$ and $\widetilde{\mathbf{S}}^{(1 / \delta)}$ are needed to satisfy $(2.10 c)$ for a quiescent spherical drop, while the terms with superscripts $(0)$ denote the Newtonian velocity, pressure and stress fields. The first non-Newtonian correction to fluid motion inside and outside the spherical drop occurs at $O(\lambda)$, which then represents the contribution from the two separate second-order fluids. Similarly, the first deformation corrections (with the fluids assumed to be Newtonian) are denoted by superscripts $(\delta)$. Inherent in the form of these asymptotic expansions is the possibility that the non-Newtonian fluid properties and the drop deformation (for Newtonian fluids) will each provide an independent first-order contribution to fluid motion and particle migration, with their interaction occurring only at $O(\lambda \delta)$. Obviously, the higher order non-Newtonian and deformation corrections will occur at $O\left(\lambda^{2}\right)$ and $O\left(\delta^{2}\right)$, respectively.

The shape of the drop should also be considered in the context of the expansions (2.12) and (2.13). Since the Newtonian velocity field alone is sufficient to cause deformation of a Newtonian drop at $O(\delta)$, it is obvious that the $O(\lambda)$ non-Newtonian velocity field will cause deformation at $O(\lambda \delta)$, and so on. Hence on the surface of the drop, we let

$$
F=r-1-\delta f^{(\delta)}-\lambda \delta f^{(\lambda \delta)}-\delta^{2} f^{(\delta \delta)}-\ldots=0,
$$

where $f^{(\delta)}, f^{(\lambda \delta)}$ and $f^{(\delta \delta)}$ denote the deformations at $O(\delta), O(\lambda \delta)$ and $O\left(\delta^{2}\right)$ respectively. Of course, it is inherently assumed from the form of (2.14) that these shape functions $f^{(\delta)}, f^{(\lambda \delta)}$ and $f^{(\delta \delta)}$ at any material point on the surface will only depend on its angular position relative to the centroid $O$ of the drop, and not on the radial position $r$ itself. The outer normal and the principal radii of curvature are now easily expressed in terms of the shape functions as

$$
\begin{aligned}
\mathbf{n}= & \nabla F /|\nabla F| \\
= & \mathbf{e}_{r}-\delta \nabla f^{(\delta)}-\lambda \delta \nabla f^{(\lambda \delta)}-\delta^{2}\left[\nabla f^{(\delta \delta)}+\frac{1}{2}\left(\nabla f^{(\delta)} \cdot \nabla f^{(\delta)}\right) \mathbf{e}_{r}\right]-\ldots \\
\frac{1}{R_{1}}+\frac{1}{R_{2}}= & \nabla \cdot \mathrm{n}=2-\delta\left[2 f^{(\delta)}+\nabla^{2} f^{(\delta)}\right]-\lambda \delta\left[2 f^{(\lambda \delta)}+\nabla^{2} f^{(\lambda \delta)}\right] \\
& -\delta^{2}\left[2 f^{(\delta \delta)}-2 f^{(\delta)} f^{(\delta)}+\nabla^{2} f^{(\delta \delta)}\right]-\ldots
\end{aligned}
$$

We now substitute (2.12), (2.13), (2.15) and (2.16) into boundary conditions (2.10a)$(2.10 \mathrm{c})$. Any quantities that are to be evaluated on the surface of the drop will be approximated by a Taylor's series expansion about $r=1$ using (2.14). Hence we have, in effect, reduced the problem to that of a spherical drop. After some straightforward algebra, velocity, kinematic and stress conditions at $r=1$ may be obtained at each order of our perturbation expansion. We shall present these equations as we need them in subsequent sections.

It should be emphasized here that a full solution of the above problem at any order in $\lambda$ or $\delta$ will yield an expression at the same level in $\lambda$ or $\delta$ for the cross-flow migration velocity. However, at any point beyond the initial Newtonian velocity fields for a spherical drop, the required analysis becomes exceedingly tedious and subject to uncertainty in the numerical accuracy of the many algebraic manipulations. Thus, in the next section, we describe the development of a theoretical expression for the lateral velocity of a deformed drop which can be evaluated at any order in $\lambda$ or $\delta$, using only the velocity and pressure corrections in the fluid at one order less in $\lambda$ or $\delta$ (i.e. at $O(1)$ for the $O(\lambda)$ and $O(\delta)$ contributions to the migration velocity). 


\section{The reciprocal theorem}

For problems in which perturbation expansions are used to extend results beyond the domain of zero Reynolds number for a particle of fixed shape in a Newtonian fluid, it is often possible to obtain, by use of the reciprocal theorem of Lorentz, macroscopic properties of interest (e.g. force and torque) at any order, without detailed calculations of the velocity field at that order; instead, only lower order solutions are needed for the velocity and pressure fields. This approach was outlined by Cox \& Brenner (1968) in connexion with the problem of inertia-induced migration of a rigid sphere. Later, Ho \& Leal $(1974,1976)$ also used the same method for their inertial and non-Newtonian migration calculations. So far, however, no one has applied the theorem to the case of a fluid drop. Since the derivation of the theorem is an important (and interesting) part in the development of our analysis, we shall present it in detail here, even though the rigid sphere problem has been treated thoroughly by previous authors.

To apply the reciprocal theorem to the calculation of lateral migration velocities, we must first consider the 'so-called' complementary problem of the motion of a Newtonian drop translating perpendicularly to the walls in a quiescent Newtonian fluid. The equations of motion outside the drop are simply

$$
\nabla . \mathbf{t}=\mathbf{0}, \quad \nabla . \mathbf{u}=\mathbf{0},
$$

where

$$
\mathbf{t}=-q \mathbf{l}+\mathbf{a}
$$

$\mathbf{a}$ is, of course, the rate-of-strain tensor. Similar equations are satisfied by $(\tilde{\mathbf{u}}, \tilde{q}, \tilde{\mathbf{t}})$ inside the drop. The boundary conditions at large distances are

$$
\left.\begin{array}{ll}
\mathbf{u} \rightarrow-\mathbf{e}_{3} & \text { as } r \rightarrow \infty, \\
\mathbf{u}=-\mathbf{e}_{3} & \text { on the walls. }
\end{array}\right\}
$$

The shape of the drop and the boundary conditions on its surface remain to be discussed. In general, it is necessary to assume that the particle in the complementary problem has the same shape as the 'real' one under consideration. However, in $\$ 2$, we have effectively reduced the full problem to that of a spherical drop with a series of boundary conditions on the surface. Hence, we may now conveniently choose the drop for the complementary problem to be also spherical, so that the boundary conditions on $r=1$ become

$$
\begin{gathered}
\mathbf{u}=\tilde{\mathbf{u}}, \\
\mathbf{u} \cdot \mathbf{e}_{r}=\tilde{\mathbf{u}} \cdot \mathbf{e}_{r}=0 \\
\left(\mathbf{I}-\mathbf{e}_{r} \mathbf{e}_{r}\right) \cdot\left(\mathbf{t} \cdot \mathbf{e}_{r}\right)=\kappa\left(\mathbf{I}-\mathbf{e}_{r} \mathbf{e}_{r}\right) \cdot\left(\tilde{\mathbf{t}} \cdot \mathbf{e}_{r}\right) .
\end{gathered}
$$

We see from (3.4c) that only shear stresses can be matched in our present problem of an undeformed sphere. Hence, a discontinuity in normal stress will usually exist on the sphere surface. (Equivalently, we may imagine that a force is being applied in the normal direction to prevent the sphere from deforming.) This discontinuity vanishes only for the well-known case of translation in an unbounded fluid medium, and even in this case, it is non-zero if wall reflexions are included.

We are now ready to apply the reciprocal theorem. For the fluid outside the drop, 
it is desirable to use disturbance quantities which approach zero far away from the particle. Therefore, we write

$$
\int_{V_{f}}\left[(\nabla \cdot \mathbf{S}-\nabla \cdot \mathbf{T}) \cdot\left(\mathbf{u}+\mathbf{e}_{3}\right)-(\nabla \cdot \mathbf{t}) \cdot(\mathbf{U}-\mathbf{V})\right] d V=0,
$$

where $V_{f}$ is the entire volume outside the drop. Rearranging, we get

$$
\int_{V_{f}} \nabla \cdot\left[(\mathbf{S}-\mathbf{T}) \cdot\left(\mathbf{u}+\mathbf{e}_{3}\right)-\mathbf{t} \cdot(\mathbf{U}-\mathbf{V})\right] d V=\int_{V_{f}}[(\mathbf{S}-\mathbf{T}): \nabla \mathbf{u}-\mathbf{t}: \nabla(\mathbf{U}-\mathbf{V})] d V .
$$

The second integral may now be easily simplified using the definitions of $\mathbf{S}, \mathbf{T}$ and $\mathbf{t}$, and the continuity equation. For the first integral, we apply the divergence theorem to obtain $\dagger$

$$
\int_{V_{f}} \nabla \cdot\left[(\mathbf{S}-\mathbf{T}) \cdot\left(\mathbf{u}+\mathbf{e}_{3}\right)-\mathbf{t} \cdot(\mathbf{U}-\mathbf{V})\right] d V=-\int_{A_{d}}\left[(\mathbf{S}-\mathbf{T}) \cdot\left(\mathbf{u}+\mathbf{e}_{3}\right)-\mathbf{t} \cdot(\mathbf{U}-\mathbf{V})\right] \cdot \mathbf{n} d A
$$

where $A_{d}$ is the spherical drop interface. Hence, for a neutrally buoyant drop

$$
\begin{aligned}
& \text { (i.e. } \left.\int_{A_{d}}(\mathbf{S}-\mathbf{T}) \cdot \mathbf{n} d A=0\right) \text {, } \\
& -\int_{A_{d}}[(\mathbf{S}-\mathbf{T}) \cdot \mathbf{u}-\mathbf{t} \cdot(\mathbf{U}-\mathbf{V})] \cdot \mathbf{n} d A \\
& =\lambda \int_{V_{f}}\left[\left(\mathbf{D}_{(1)} \cdot \mathbf{D}_{(1)}-\mathbf{E}_{(\mathbf{1})} \cdot \mathbf{E}_{(1)}\right)+\epsilon_{1}\left(\mathbf{D}_{(2)}-\mathbf{E}_{(2)}\right)\right]: \nabla \mathbf{u} d V \\
& +\lambda^{2} \int_{V_{f}}\left[\epsilon_{2}, \epsilon_{3}, \epsilon_{4} \text { terms }\right]: \nabla \mathbf{u} d V \text {. }
\end{aligned}
$$

Inside the drop, similar manipulations give

$$
\int_{A_{d}}[\tilde{\mathbf{S}} \cdot \tilde{\mathbf{u}}-\tilde{\mathbf{t}} \cdot \tilde{\mathbf{U}}] \cdot \mathbf{n} d A=\tilde{\lambda} \int_{\tilde{V}_{f}}\left[\widetilde{\mathbf{D}}_{(1)} \cdot \widetilde{\mathbf{D}}_{(1)}+\tilde{\epsilon}_{1} \widetilde{\mathbf{D}}_{(2)}\right]: \nabla \tilde{\mathbf{u}} d V+\tilde{\lambda}^{2} \int_{\widetilde{V}_{f}}\left[\tilde{\epsilon}_{2}, \tilde{\epsilon}_{3}, \tilde{\epsilon}_{4} \text { terms }\right]: \nabla \tilde{\mathbf{u}} d V
$$

with $\tilde{V}_{f}$ denoting the volume of the drop. We now have two expressions (3.8) and (3.9), which both involve area integrals on the surface of the drop. To evaluate them, we need to consider the boundary conditions $(2.10)$ at the surface of the drop, transformed to apply at $r=1$. These conditions are of the matching type; hence, it is obvious that (3.8) and (3.9) can be combined by multiplying (3.9) by $\kappa$ and adding it to (3.8). The result is

$$
\begin{aligned}
&-\int_{A_{d}}[(\mathbf{S}-\kappa \tilde{\mathbf{S}}) \cdot \mathbf{u}-(\mathbf{t}-\kappa \tilde{\mathbf{t}}) \cdot \mathbf{U}-\kappa \tilde{\mathbf{t}} \cdot(\mathbf{U}-\tilde{\mathbf{U}})-\mathbf{T} \cdot \mathbf{u}+\mathbf{t} \cdot \mathbf{V}] \cdot \mathbf{n} d A \\
&= \lambda\left\{\int_{V_{f}}\left[\left(\mathbf{D}_{(\mathbf{1})} \cdot \mathbf{D}_{(\mathbf{1})}-\mathbf{E}_{(\mathbf{1})} \cdot \mathbf{E}_{(1)}\right)+\epsilon_{1}\left(\mathbf{D}_{(2)}-\mathbf{E}_{(2)}\right)\right]: \nabla \mathbf{u} d V\right. \\
&\left.+\kappa \frac{\bar{\lambda}}{\lambda} \int_{\tilde{V}_{f}}\left[\tilde{\mathbf{D}}_{(\mathbf{1})} \cdot \tilde{\mathbf{D}}_{(1)}+\tilde{\epsilon}_{1} \tilde{\mathbf{D}}_{(2)}\right]: \nabla \tilde{\mathbf{u}} d V\right\}+\lambda^{2}\left\{\int_{V_{f}}\left[\epsilon_{2}, \epsilon_{3}, \epsilon_{4} \text { terms }\right]: \nabla \mathbf{u} d V\right. \\
&\left.+\kappa(\tilde{\lambda} / \lambda)^{2} \int_{\tilde{V}_{f}}\left[\tilde{\epsilon}_{2}, \tilde{\epsilon}_{3}, \tilde{\epsilon}_{4} \text { terms }\right]: \nabla \tilde{\mathbf{u}} d V\right\} .
\end{aligned}
$$

† It may be shown, for disturbance quantities which approach zero at large distances from the particle, that it is not necessary to consider the contributions at infinity. A detailed proof is provided in Chan (1979). 
The advantage of combining (3.8) and (3.9) in the form (3.10) is that we can use the interface boundary conditions to evaluate the surface integral over $A_{a}$ to $O\left(\lambda^{n}\right)$ in terms of the migration velocity contribution at $O\left(\lambda^{n}\right)$ and the fluid velocity and stress fields through $O\left(\lambda^{n-1}\right)$. It may be noted, in this regard, that the drop shape is quasisteady at $O\left(\delta^{m}\right)$ and can thus be determined via the normal stress condition at $O\left(\delta^{m}\right)$ from the stress fields at $O\left(\delta^{m-1}\right)$. This latter fact is obviously crucial to the successful evaluation of the left-hand side of (3.10) in the manner described above. Since the right-hand side of (3.10) may obviously also be calculated at $O\left(\lambda^{n}\right)$ completely in terms of the velocity and stress fields at $O\left(\lambda^{n-1}\right)$, the expression (3.10) can clearly be used to determine the migration velocity at $O\left(\lambda^{n}\right)$ completely in terms of known or calculable quantities at $O\left(\lambda^{n-1}\right)$. This fact may, perhaps, be more clearly illustrated by considering the $O(\lambda)$ terms in (3.10)

$$
\begin{gathered}
-\int_{A_{d}}\left[\left(\mathbf{S}^{(\lambda)}-\kappa \tilde{\mathbf{S}}^{(\lambda)}\right) \cdot \mathbf{u}-(\mathbf{t}-\kappa \tilde{\mathbf{t}}) \cdot \mathbf{U}^{(\lambda)}-\kappa \tilde{\mathbf{t}} \cdot\left(\mathbf{U}^{(\lambda)}-\tilde{\mathbf{U}}^{(\lambda)}\right)-\mathbf{T}^{(\lambda)} \cdot \mathbf{u}+\mathbf{t} \cdot \mathbf{V}^{(\lambda)}\right] \cdot \mathbf{e}_{r} d A \\
=\int_{V_{f}}\left[\left(\mathbf{D}_{(1)}^{(0)} \cdot \mathbf{D}_{(1)}^{(0)}-\mathbf{E}_{(1)}^{(0)} \cdot \mathbf{E}_{(1)}^{(0)}\right)+\epsilon_{\mathbf{1}}\left(\mathbf{D}_{(2)}^{(0)}-\mathbf{E}_{(2)}^{(0)}\right)\right]: \nabla \mathbf{u} d V \\
\quad+\kappa \frac{\tilde{\lambda}}{\lambda} \int_{\tilde{V}_{f}}\left[\tilde{\mathbf{D}}_{(1)}^{(0)} \cdot \tilde{\mathbf{D}}_{(1)}^{(0)}+\tilde{\epsilon}_{\mathbf{1}} \tilde{\mathbf{D}}_{(2)}^{(0)}\right]: \nabla \tilde{\mathbf{u}} d V
\end{gathered}
$$

As we shall show in $\S 5$, the surface integral over $A_{d}$ can be easily simplified using the interfacial boundary conditions so that only Newtonian velocity fields appear from $\mathbf{T}^{(\lambda)}$. u. $\mathbf{e}_{r}$, along with the migration velocity $\hat{\mathbf{U}}_{s}^{(\lambda)}$ which comes from $\mathbf{t} . \mathbf{V}^{(\lambda)} \cdot \mathbf{e}_{r}$. Obviously, expressions for $\hat{\mathbf{U}}_{s}^{(\delta)}$ or the higher order quadratic terms can be obtained in a similar manner, and these will be presented as needed in later sections of the paper.

\section{The Newtonian solutions}

We now attempt to solve, to $O(1)$, the equations of motion (2.1) and (2.4), subject to boundary conditions (2.9) and $(2.10 a)-(2.10 c)$. As before, the superscript $(1 / \delta)$ will denote the pressure term at quiescence, whereas the superscript $(0)$ denotes the Newtonian contributions. Trivially, we obtain

$$
\breve{P}^{(1 / \delta)}=2 / \kappa .
$$

It is obvious that this term represents the capillary pressure increase across the surface of a spherical drop in a quiescent fluid.

The Newtonian flow problem outside the drop is defined by

$$
\nabla \cdot \mathbf{S}^{(0)}=0, \quad \nabla \cdot \mathbf{U}^{(0)}=0
$$

with boundary conditions at large distances from the drop

$$
\begin{aligned}
& \mathbf{U}^{(0)} \rightarrow\left(\alpha+\beta x_{3}+\gamma x_{3}^{2}\right) \mathbf{e}_{1}-\hat{\mathbf{U}}_{s}^{(0)} \quad \text { as } r \rightarrow \infty, \\
& \mathbf{U}^{(0)}=V_{w} \mathbf{e}_{1}-\hat{\mathbf{U}}_{s}^{(0)} \quad \text { on the walls. }
\end{aligned}
$$

It is, of course, very difficult to obtain an exact analy tic solution to the above boundary value problem. However, for a particle which is small compared to the characteristic dimension of the flow (i.e. $\zeta \ll 1$ ), we may utilize the well-known method of reflexions (cf. Happel \& Brenner 1973), which approximates $U^{(0)}$ as a series of terms alternately 
satisfying the boundary conditions at the particle surface and on the walls. Inherently, it is assumed in this technique that the particle is not 'close' to either wall. For our case, we write

$$
\mathbf{U}^{(0)}={ }_{1} \mathbf{U}^{(0)}+{ }_{2} \mathbf{U}^{(0)}+{ }_{3} \mathbf{U}^{(0)}+\ldots
$$

The solution of the above equations follows the method outlined by Ho \& Leal (1974) for a rigid sphere. The first term in (4.4) corresponds to the velocity in an unbounded domain and is obtained using Lamb's general solution,

$$
\begin{aligned}
{ }_{1} \mathbf{U}^{(0)}= & \left(\alpha+\beta x_{3}+\gamma x_{3}^{2}\right) \mathbf{e}_{1}-\hat{\mathbf{U}}_{s}^{(0)}-\frac{1}{2} A_{1}\left(\frac{1}{r} \mathbf{e}_{1}+\frac{x_{1}}{r^{3}} \mathbf{x}\right)-B_{1}\left(\frac{1}{r^{3}} \mathbf{e}_{1}-\frac{3 x_{1}}{r^{5}} \mathbf{x}\right) \\
& -C_{1}\left(\frac{x_{3}}{r^{3}} \mathbf{e}_{1}-\frac{x_{1}}{r^{3}} \mathbf{e}_{3}\right)+\frac{3}{2} D_{1} \frac{x_{1} x_{3}}{r^{5}} \mathbf{x}+3 E_{1}\left(\frac{x_{3}}{r^{5}} \mathbf{e}_{1}+\frac{x_{1}}{r^{5}} \mathbf{e}_{3}-\frac{5 x_{1} x_{3}}{r^{7}} \mathbf{x}\right) \\
& -3 F_{1}\left(\frac{1}{r^{3}} \mathbf{e}_{1}-\frac{x_{1}}{r^{5}} \mathbf{x}-\frac{2 x_{3}^{2}}{r^{5}} \mathbf{e}_{1}+\frac{2 x_{1} x_{3}}{r^{5}} \mathbf{e}_{3}\right) \\
& -\frac{1}{10} G_{1}\left(\frac{1}{r^{3}} \mathbf{e}_{1}-\frac{13 x_{1}}{r^{5}} \mathbf{x}-\frac{5 x_{3}^{2}}{r^{5}} \mathbf{e}_{1}-\frac{10 x_{1} x_{3}}{r^{5}} \mathbf{e}_{3}+\frac{75 x_{1} x_{3}^{2}}{r^{7}} \mathbf{x}\right) \\
& +3 H_{1}\left(\frac{1}{r^{5}} \mathbf{e}_{1}-\frac{5 x_{1}}{r^{7}} \mathbf{x}-\frac{5 x_{3}^{2}}{r^{7}} \mathbf{e}_{1}-\frac{10 x_{1} x_{3}}{r^{7}} \mathbf{e}_{3}+\frac{35 x_{1} x_{3}^{2}}{r^{9}} \mathbf{x}\right) .
\end{aligned}
$$

By considering (4.5), the first reflected solution ${ }_{2} \mathbf{U}^{(0)}$ may now be expressed in terms of complicated integrals over the entire volume (i.e. Faxén's method; cf. Happel \& Brenner 1973). Here, we consider only the simplified form which is relevant in the vicinity of the drop

$$
{ }_{2} \mathbf{U}^{(0)}=\left(I_{1}+\frac{1}{2} I_{4}\right) \mathbf{e}_{1}-\frac{1}{2} \zeta\left(\frac{3}{2} I_{2}+I_{5}\right)\left(x_{3} \mathbf{e}_{1}+x_{1} \mathbf{e}_{3}\right)-\frac{1}{2} \zeta\left(\frac{1}{2} I_{2}-I_{7}\right)\left(x_{3} \mathbf{e}_{1}-x_{1} \mathbf{e}_{3}\right) .
$$

For brevity, we refer the reader to Ho \& Leal (1974) for the detailed expressions for $I_{1} \ldots I_{7}$; they do not appear in any final results of this paper. Finally, proceeding from (4.6), we obtain ${ }_{3} U^{(0)}$ in the form

$$
\begin{aligned}
{ }_{3} \mathbf{U}^{(0)}= & -\frac{1}{2} A_{3}\left(\frac{1}{r} \mathbf{e}_{1}+\frac{x_{1}}{r^{3}} \mathbf{x}\right)-B_{3}\left(\frac{1}{r^{3}} \mathbf{e}_{1}-\frac{3 x_{1}}{r^{5}} \mathbf{x}\right)-C_{3}\left(\frac{x_{3}}{r^{3}} \mathbf{e}_{1}-\frac{x_{1}}{r^{3}} \mathbf{e}_{3}\right) \\
& +\frac{3}{2} D_{3} \frac{x_{1} x_{3}}{r^{5}} \mathbf{x}+3 E_{3}\left(\frac{x_{3}}{r^{5}} \mathbf{e}_{1}+\frac{x_{1}}{r^{5}} \mathbf{e}_{3}-\frac{5 x_{1} x_{3}}{r^{7}} \mathbf{x}\right) .
\end{aligned}
$$

For the fluid inside the drop, we let

$$
\widetilde{U}^{(0)}={ }_{1} \widetilde{\mathbf{U}}^{(0)}+{ }_{2+3} \widetilde{\mathrm{U}}^{(0)}+\ldots,
$$

where ${ }_{2+3} \tilde{U}^{(0)}$ is the term needed to match ${ }_{2} U^{(0)}+{ }_{3} U^{(0)}$ at the drop surface. Again, using Lamb's general solution, we obtain

$$
\begin{aligned}
\widetilde{\mathbf{U}}^{(0)}= & -\frac{1}{10} \widetilde{A}_{1}\left(2 r^{2} \mathbf{e}_{1}-x_{1} \mathbf{x}\right)-\widetilde{B}_{1} \mathbf{e}_{1}-\tilde{C}_{1}\left(x_{3} \mathbf{e}_{1}-x_{1} \mathbf{e}_{3}\right) \\
& +\frac{1}{14} \tilde{D}_{1}\left(5 x_{3} r^{2} \mathbf{e}_{1}+5 x_{1} r^{2} \mathbf{e}_{3}-4 x_{1} x_{3} \mathbf{x}\right)-3 \tilde{E}_{1}\left(x_{3} \mathbf{e}_{1}+x_{1} \mathbf{e}_{3}\right) \\
& -3 \tilde{F}_{1}\left(r^{2} \mathbf{e}_{1}-x_{1} \mathbf{x}-2 x_{3}^{2} \mathbf{e}_{1}+2 x_{1} x_{3} \mathbf{e}_{3}\right)+\frac{1}{4} \widetilde{G}_{1}\left(r^{4} \mathbf{e}_{1}+x_{1} r^{2} \mathbf{x}-5 x_{3}^{2} r^{2} \mathbf{e}_{1}\right. \\
& \left.-10 x_{1} x_{3} r^{2} \mathbf{e}_{3}+5 x_{1} x_{3}^{2} \mathbf{x}\right)+3 \tilde{H}_{1}\left(r^{2} \mathbf{e}_{1}+2 x_{1} \mathbf{x}-5 x_{3}^{2} \mathbf{e}_{1}-10 x_{1} x_{3} \mathbf{e}_{3}\right) .
\end{aligned}
$$

The solution ${ }_{2+3} \widetilde{\mathrm{U}}^{(0)}$ is similar to $(4.9)$ but with $\tilde{A}_{1} \ldots \widetilde{E}_{1}$ replaced by $\tilde{A}_{3} \ldots \widetilde{E}_{3}$, while the corresponding $\tilde{F}_{3} \ldots \tilde{H}_{3}$ terms may be omitted at this level of approximation. 
All the constants $A_{1} \ldots H_{1}, A_{3} \ldots E_{3}, \tilde{A}_{1} \ldots \tilde{H}_{1}$ and $\tilde{A}_{3} \ldots \tilde{E}_{3}$, and the unknown shape function $f^{(\delta)}$ are determined via application of the boundary conditions $(2.10 a)$ $(2.10 c)$ at $r=1$. Using (2.15) and (2.16), these conditions are

$$
\begin{gathered}
\mathbf{U}^{(0)}=\tilde{\mathbf{U}}^{(0)}, \\
\mathbf{U}^{(0)} \cdot \mathbf{e}_{r}=\tilde{\mathbf{U}^{(0)}} \cdot \mathbf{e}_{r}=0, \\
\mathbf{S}^{(0)} \cdot \mathbf{e}_{r}=\kappa \tilde{\mathbf{S}}^{(0)} \cdot \mathbf{e}_{r}-\left[2 f^{(\delta)}+\nabla^{2} f^{(\delta)}\right] \mathbf{e}_{r} .
\end{gathered}
$$

After some algebra, the coefficients in (4.5), (4.7) and (4.9) are obtained from (4.10a), $(4.10 b)$, and the tangential component of $(4.10 c)$

$$
\begin{array}{ll}
A_{1}=\left[\alpha-\left(\hat{U}_{s}^{(0)}\right)_{1}\right] \frac{2+3 \kappa}{2(1+\kappa)}+\frac{\gamma \kappa}{2(1+\kappa)}, & \tilde{A}_{1}=-\left[\alpha-\left(\hat{U}_{s}^{(0)}\right)_{1}\right] \frac{5}{1+\kappa}-\frac{5 \gamma}{1+\kappa}, \\
B_{1}=\left[\alpha-\left(\hat{U}_{s}^{(0)}\right)_{1}\right] \frac{\kappa}{4(1+\kappa)}+\frac{\gamma(-2+3 \kappa)}{20(1+\kappa)}, & \tilde{B}_{1}=\left[\alpha-\left(\hat{U}_{s}^{(0)}\right)_{1}\right] \frac{1}{2(1+\kappa)}+\frac{\gamma}{2(1+\kappa)}, \\
C_{1}=0, & \tilde{C}_{1}=-\frac{1}{2} \beta, \\
D_{1}=-\frac{\beta(2+5 \kappa)}{3(1+\kappa)}, & \tilde{D}_{1}=\frac{7 \beta}{2(1+\kappa)}, \\
E_{1}=-\frac{\beta \kappa}{6(1+\kappa)}, & \widetilde{E}_{1}=-\frac{\beta}{4(1+\kappa)}, \\
F_{1}=\frac{\gamma(1-\kappa)}{9(4+\kappa)}, & \tilde{F}_{1}=\frac{5 \gamma}{9(4+\kappa)}, \\
G_{1}=\frac{\gamma(2+7 \kappa)}{12(1+\kappa)}, & \tilde{G}_{1}=-\frac{\gamma}{1+\kappa}, \\
H_{1}=\frac{\gamma \kappa}{24(1+\kappa)}, & \tilde{H}_{1}=\frac{\gamma}{18(1+\kappa)} ;
\end{array}
$$

It is easy to see that the velocity fields (4.4) and (4.8), together with the coefficients (4.11) and (4.12), reduce to the values given by Ho \& Leal (1974) for the case of a very viscous drop (i.e. $\kappa \rightarrow \infty$ ). In this limit the motion of the drop reduces to a rigid body rotation, as expected.

The force acting on the drop, to our present level of approximation, may be obtained by summing the Stokeslet contributions from (4.5) and (4.7), which gives

$$
\mathbf{F}=4 \pi\left(A_{1}+A_{3}\right) \mathbf{e}_{1} .
$$

Obviously, for a neutrally buoyant particle, the force $\mathbf{F}$ must be zero, and it thus follows from (4.13) that

$$
\left(\hat{U}_{s}^{(0)}\right)_{1}=\alpha+\kappa \gamma /(2+3 \kappa)+I_{1}+\frac{1}{2} I_{4}
$$



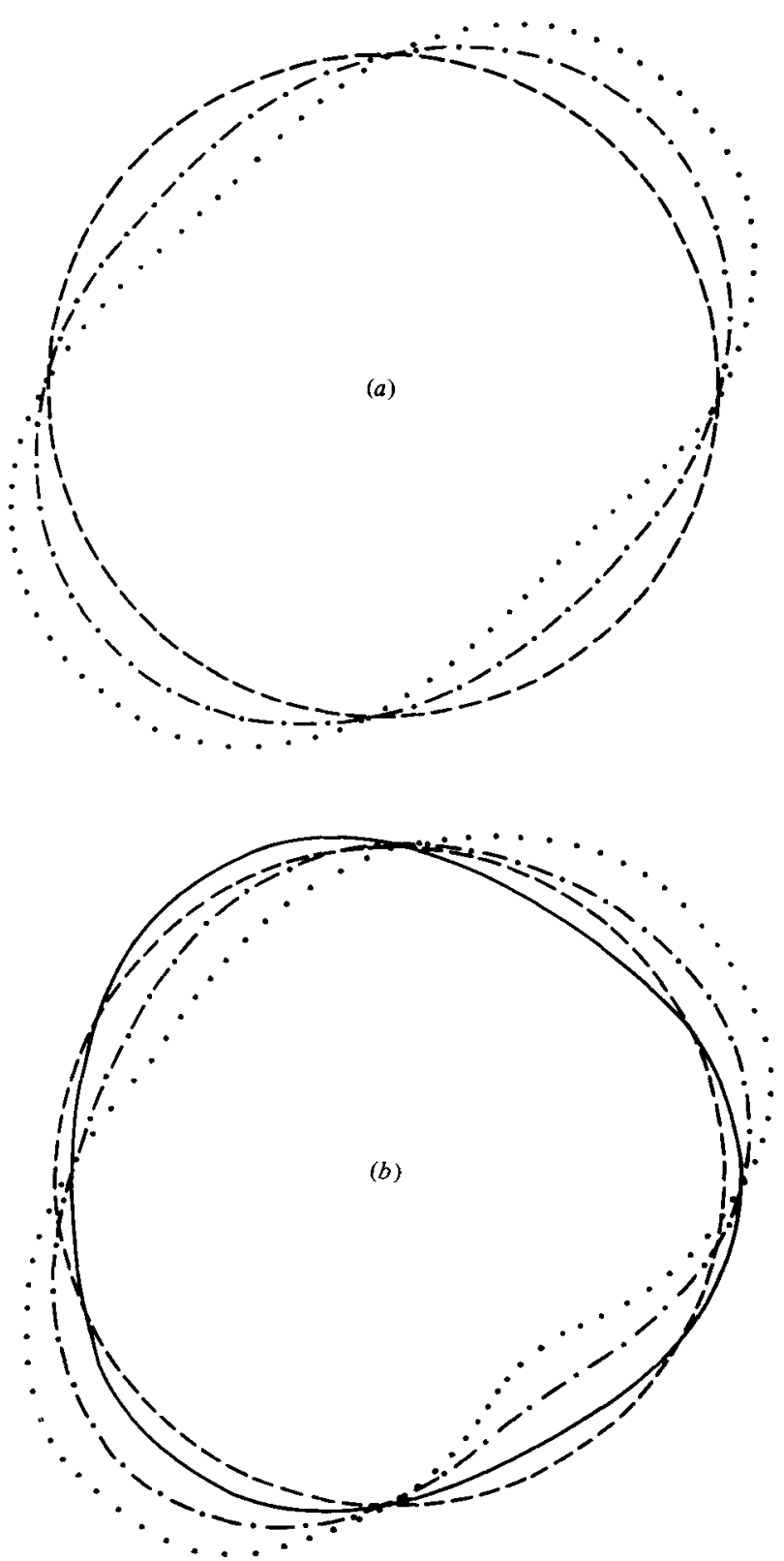

Frgure 2. The shape of a deformed drop in: $(a)$ a simple shear flow. $-\cdot-, \chi=\delta \beta(16+19 \kappa) /$ $8(1+\kappa)=0.25 ; \cdots, \chi=0.50 ;(b)$ a two-dimensional quadratic shearing flow $(-\delta \gamma(10+11 \kappa) /$ $40(1+\kappa)=0.05$ ). $\longrightarrow, \chi=0$ (i.e. centre-line for Poiseuille flow); -,$- \chi=0.25 ; \cdots, \chi=0.50$; ,-- no deformation (i.e. $r=1$ ).

in this case. For a plane Poiseuille flow, the dominant contribution to the particle velocity relative to the local undisturbed flow (i.e. $\left.\left(\hat{U}_{s}^{(0)}\right)_{1}-\alpha\right)$ comes from the shear gradient $\gamma$, which is negative for all values of $s$. Thus, the drop will always lag behind the surrounding fluid. For a simple shear flow, $\gamma$ is identically zero; in this case, the slip velocity can only arise from wall reflexions. By Ho \& Leal (1974), $I_{1}+\frac{1}{2} I_{4}$ itself 
depends on $\beta$ and changes sign at $s=0 \cdot 5$. As a result, in a simple shear flow, the drop leads the fluid for $s>0.5$ but lags behind it for $s<0.5$.

The shape of the drop can now be obtained, using the normal stress term of $(4.10 \mathrm{c})$. To this end, we assume that the volume of the drop remains constant, and that its centroid coincides with origin $O$ of our co-ordinate system. Then, expressing $f^{(\delta)}$ in the form

we obtain

$$
f^{(\delta)}={ }_{1} f^{(\delta)}+{ }_{2+3} f^{(\delta)}+\ldots
$$

It can be seen easily that $(4.16 a)$ agrees exactly with the result of Taylor $(1932,1934)$ for a simple, unbounded shear flow $(\gamma=0)$, and also with Haber \& Hetrsoni (1971) for the case of an unbounded quadratic shearing flow. On the other hand, $(4.16 b)$ represents an additional deformation of the drop due to hydrodynamic interaction with the walls. Its form is identical to that for a drop in an unbounded linear shear flow, and its magnitude relative to the first term of $(4.16 a)$ is $-(\zeta / \beta) \times\left(\frac{3}{2} I_{2}+I_{5}\right)$. This function is of $O\left(\zeta^{3}\right)$ and can be calculated numerically following Ho \& Leal (1974). As expected, it is symmetric about $s=0.5$, and is positive for all values of $s$. Thus, the drop deformation is always increased by the presence of the walls. Using $\zeta=0.1$ and $\kappa=0$, we calculate $-(\zeta / \beta) \times\left(\frac{3}{2} I_{2}+I_{5}\right)$ to be 0.0057 at $s=0.5$ (i.e. centre-line), 0.015 at $s=0.3$, and 0.048 at $s=0.2$ (i.e. two drop radii from wall). Hence, its contribution is significant only when the drop is very near to a wall. In figure $2(a)$ and $(b)$, we plot the drop shape in the cases of a simple shear flow and a two-dimensional quadratic shearing flow.

To apply the Newtonian solution to migration calculations in the next two sections, we need also to obtain the complementary velocity fields $\mathbf{u}$ and $\tilde{\mathbf{u}}$ defined in $\S 3$. As above, they are expanded as

and

$$
\begin{aligned}
& \mathbf{u}={ }_{1} \mathbf{u}+{ }_{2} \mathbf{u}+{ }_{3} \mathbf{u}+\ldots \\
& \tilde{\mathbf{u}}={ }_{1} \tilde{\mathbf{u}}+{ }_{2+3} \tilde{\mathbf{u}}+\ldots
\end{aligned}
$$

The unbounded domain solutions are

$$
\begin{aligned}
& { }_{1} \mathbf{u}=-\mathbf{e}_{3}-\frac{1}{2} a_{1}\left(\frac{1}{r} \mathbf{e}_{3}+\frac{x_{3}}{r^{3}} \mathbf{x}\right)-b_{1}\left(\frac{1}{r^{3}} \mathbf{e}_{3}-\frac{3 x_{3}}{r^{5}} \mathbf{x}\right), \\
& { }_{1} \tilde{\mathbf{u}}=-\frac{1}{10} \tilde{a}_{1}\left(2 r^{2} \mathbf{e}_{3}-x_{3} \mathbf{x}\right)-\tilde{b}_{1} \mathbf{e}_{3},
\end{aligned}
$$

where $\quad a_{1}=-\frac{2+3 \kappa}{2(1+\kappa)}, \quad \tilde{a}_{1}=\frac{5}{1+\kappa}, \quad b_{1}=-\frac{\kappa}{4(1+\kappa)}, \quad \tilde{b}_{1}=-\frac{1}{2(1+\kappa)}$.

These are, of course, identical to the well-known solution of Hadamard (1911) and Rybczynski (1911) for the motion of a spherical drop in an unbounded quiescent fluid. To obtain the reflected solutions, we again follow the procedures outlined by Ho \& Leal (1974) ${ }_{2} \mathbf{u}$ is expressed in terms of complicated integrals, but simplifies near the drop to the form

$$
{ }_{2} \mathbf{u}=\left[-\left(J_{1}+J_{4}\right)+\frac{3}{2} \zeta J_{2} x_{3}\right] \mathbf{e}_{3}-\frac{1}{2} \zeta J_{2} \mathbf{x}
$$


The term $J_{2}$ will appear repeatedly in our results. Hence, we note its detailed form here

$$
\begin{aligned}
J_{2}=\frac{\zeta}{32} \frac{2+3 \kappa}{1+\kappa} \int_{0}^{\infty} \frac{\xi^{3}}{\left(1-e^{-\xi}\right)^{2}-\xi^{2} e^{-\xi}}\left\{s^{2}\left[e^{-8 \xi}+e^{-(2-8) \xi}\right]\right. \\
\left.-(1-s)^{2}\left[e^{-(1-s) \xi}+e^{-(1+s) \xi}\right]+2(1-2 s) e^{-\xi}\right\} d \xi .
\end{aligned}
$$

In general, this integral has to be evaluated numerically for any given value of $s$. However, an excellent approximation (with less than $2 \%$ error for all $s$ ) can be obtained by simply substituting the integrand with

$$
\xi^{3}\left[s^{2} e^{-s \xi}-(1-s)^{2} e^{-(1-s) \xi}+2(1-2 s) e^{-\xi}\right],
$$

and then integrating the expression analytically to obtain

$$
J_{2} \sim \frac{3 \zeta}{16} \frac{2+3 \kappa}{1+\kappa}\left[\frac{1}{s^{2}}-\frac{1}{(1-s)^{2}}+2(1-2 s)\right] .
$$

For convenience, we shall base all subsequent calculations upon this approximate form for $J_{2}$. Following our earlier procedures, ${ }_{3} \mathbf{u}$ and ${ }_{2+3} \tilde{\mathbf{u}}$ are now obtained from the form of ${ }_{2} \mathbf{u}$ at the drop surface. Using Lamb's general solution, we obtain

$$
\begin{aligned}
{ }_{3} \mathbf{u}= & -\frac{1}{2} a_{3}\left(\frac{1}{r} \mathbf{e}_{3}+\frac{x_{3}}{r^{3}} \mathbf{x}\right)-b_{3}\left(\frac{1}{r^{3}} \mathbf{e}_{3}-\frac{3 x_{3}}{r^{5}} \mathbf{x}\right)-\frac{1}{2} d_{3}\left(\frac{1}{r^{3}} \mathbf{x}-\frac{3 x_{3}^{2}}{r^{5}} \mathbf{x}\right) \\
& +3 e_{3}\left(\frac{1}{r^{5}} \mathbf{x}+\frac{2 x_{3}}{r^{5}} \mathbf{e}_{3}-\frac{5 x_{3}^{2}}{r^{7}} \mathbf{x}\right) . \\
{ }_{2+3} \tilde{\mathbf{u}}= & -\frac{1}{10} \tilde{a}_{3}\left(2 r^{2} \mathbf{e}_{3}-x_{3} \mathbf{x}\right)-\tilde{b}_{3} \mathbf{e}_{3}-\frac{1}{7} \tilde{d}_{3}\left(r^{2} \mathbf{x}-5 r^{2} \mathbf{e}_{3}+2 x_{3}^{2} \mathbf{x}\right)-2 \tilde{e}_{3}\left(\mathbf{x}-3 x_{3} \mathbf{e}_{3}\right) .
\end{aligned}
$$

The above equations must satisfy boundary conditions $(3.4 a)-(3.4 c)$ on $r=1$. The coefficients are therefore

$$
\left.\begin{array}{ll}
a_{3}=-\left(J_{1}+J_{4}\right)(2+3 \kappa) / 2(1+\kappa), & \tilde{a}_{3}=\left(J_{1}+J_{4}\right) 5 /(1+\kappa), \\
b_{3}=-\left(J_{1}+J_{4}\right) \kappa / 4(1+\kappa), & \tilde{b}_{3}=-\left(J_{1}+J_{4}\right) / 2(1+\kappa), \\
d_{3}=-\zeta J_{2}(2+5 \kappa) / 2(1+\kappa), & \tilde{d}_{3}=\zeta J_{2} 21 / 4(1+\kappa), \\
e_{3}=-\zeta J_{2} \kappa / 4(1+\kappa), & \tilde{e}_{3}=-\zeta J_{2} 3 / 8(1+\kappa) .
\end{array}\right\}
$$

This completes the solution for $\mathbf{u}$.

\section{The $O(\lambda)$ problem}

We now consider the $O(\lambda)$ problem of a non-Newtonian spherical drop suspended in a two-dimensional shearing flow of a second-order fluid. For the suspending phase, the equations of motion are

$$
\nabla \cdot \mathbf{S}^{(\lambda)}=0, \quad \nabla \cdot \mathbf{U}^{(\lambda)}=0,
$$

where

$$
\mathbf{S}^{(\lambda)}=-P^{(\lambda)} \mathbf{I}+\mathbf{D}_{(1)}^{(\lambda)}+\left[\mathbf{D}_{(1)}^{(0)} \cdot \mathbf{D}_{(1)}^{(0)}+\epsilon_{\mathbf{1}} \mathbf{D}_{(2)}^{(0)}\right] .
$$

The equations for the drop fluid are, of course, completely analogous to the above. At large distances from the drop, the boundary conditions are

$$
\left.\begin{array}{ll}
\mathbf{U}^{(\lambda)} \rightarrow-\hat{\mathbf{U}}_{\boldsymbol{s}}^{(\lambda)} & \text { as } r \rightarrow \infty, \\
\mathbf{U}^{(\lambda)}=-\hat{\mathbf{U}}_{\boldsymbol{s}}^{(\lambda)} & \text { on the walls, }
\end{array}\right\}
$$


whereas on $r=1$

$$
\begin{gathered}
\mathbf{U}^{(\lambda)}=\tilde{\mathbf{U}}^{(\lambda)}, \\
\mathbf{U}^{(\lambda)} \cdot \mathbf{e}_{r}=\tilde{\mathbf{U}}^{(\lambda)} \cdot \mathbf{e}_{r}=0, \\
\mathbf{S}^{(\lambda)} \cdot \mathbf{e}_{r}=\kappa \tilde{\mathbf{S}}^{(\lambda)} \cdot \mathbf{e}_{r}-\left[2 f^{(\lambda \delta)}+\nabla^{2} f^{(\lambda \delta)}\right] \mathbf{e}_{r} .
\end{gathered}
$$

It is apparent that a detailed calculation of the complete velocity fields will be extremely difficult. However, to obtain the migration velocity to $O(\lambda)$, we only need to consider (3.11) as we have noted above. From $(3.4 a)-(3.4 c)$ and $(5.4 a)-(5.4 c)$, it is obvious that the first three terms of the surface integral are all identically zero. The remaining terms simplify to

$$
\begin{aligned}
& \int_{A_{d}} \mathbf{T}^{(\lambda)} \cdot \mathbf{u} \cdot \mathbf{e}_{r} d A=-[8 \pi / 15(1+\kappa)]\left[\beta \gamma\left(1+J_{1}+J_{4}\right)-\frac{3}{4} \beta^{2} \zeta J_{2}\right]\left(1+4 \epsilon_{1}\right), \\
& \int_{A_{d}} \mathbf{t} \cdot \mathbf{V}^{(\lambda)} \cdot \mathbf{e}_{r} d A=[2 \pi(2+3 \kappa) /(1+\kappa)]\left(1+J_{1}+J_{4}\right)\left(\hat{U}_{s}^{(\lambda)}\right)_{3} .
\end{aligned}
$$

Rearranging, we thus obtain

$$
\begin{aligned}
\left(\hat{U}_{s}^{(\lambda)}\right)_{3}= & -\frac{4}{15(2+3 \kappa)}\left[\beta \gamma-\frac{3}{4} \beta^{2} \zeta_{2}\right]\left(1+4 \epsilon_{1}\right)-\frac{1+\kappa}{2 \pi(2+3 \kappa)} \cdot\left\{\int _ { V _ { f } } \left[\left(\mathbf{D}_{(1)}^{(0)} \cdot \mathbf{D}_{(1)}^{(0)}-\mathbf{E}_{(1)}^{(0)} \cdot \mathbf{E}_{(1)}^{(0)}\right)\right.\right. \\
& \left.\left.+\epsilon_{1}\left(\mathbf{D}_{(2)}^{(0)}-\mathbf{E}_{(2)}^{(0)}\right)\right]: \nabla \mathbf{u} d V+\kappa \frac{\tilde{\lambda}}{\lambda} \int_{\widetilde{V}_{f}}\left[\widetilde{\mathbf{D}}_{(1)}^{(0)} \cdot \widetilde{\mathbf{D}}_{(1)}^{(0)}+\tilde{\epsilon}_{1} \tilde{\mathbf{D}}_{(2)}^{(0)}\right]: \nabla \mathbf{u} d V\right\} .
\end{aligned}
$$

Here $J_{2} /\left(1+J_{1}+J_{4}\right)$ is approximated as simply $J_{2}$, since $J_{1}+J_{4}$ is itself of $O(\zeta)$.

We note that an exact, direct calculation of the first integral in (5.7) over the entire volume $V_{f}$ outside the drop is extremely complicated due to the presence of the bounding walls. Instead, we obtain an approximation to this term by dividing $V_{f}$ into a 'near-field' region $V_{1}$ and a 'far-field' region $V_{2}$, in a manner asymptotically consistent with the expansion in $\zeta$ which is inherent in the reflexions procedure. $V_{1}$ thus corresponds to an unbounded domain with the drop immersed in it (i.e. $1 \leqslant r<\infty$ ), whereas $V_{2}$ includes the walls while seeing the drop as merely a point (i.e. $0 \leqslant \zeta r<\infty$, $\left.-s \leqslant \zeta x_{3} \leqslant 1-s\right)$. For any particular flow at infinity, the order of magnitude of the integrands may then be obtained using the estimates for $\left({ }_{1} \mathbf{u},{ }_{2} \mathbf{u},{ }_{3} \mathbf{u}\right)$ and $\left({ }_{1} \mathbf{U}^{(0)}{ }_{2} \mathbf{U}^{(0)}\right.$, $\left.{ }_{3} \mathrm{U}^{(0)}\right)$ in $V_{1}$ and $V_{2}$ that were provided by Ho \& Leal (1974). Only the asymptotically dominant contribution needs to be evaluated. We now consider two specific cases of interest.

\subsection{The quadratic unidirectional shear flow}

Here, we consider the migration of a spherical drop in a two-dimensional quadratic unidirectional shear flow (e.g. plane Poiseuille flow). The calculation follows the general procedures that were outlined by Ho \& Leal (1976) for a rigid sphere. For the suspending phase, it can be shown that the dominant contribution to the integral over $V_{f}$ in (5.7) comes from the $\beta \gamma$ term in $V_{1}$, and is therefore of $O\left(\zeta^{3}\right)$. For the drop fluid, on the other hand, the volume integral over $\widetilde{V}_{f}$ can be evaluated directly, without approximation. Hence, using ${ }_{1} U^{(0)}, \widetilde{U}_{1}^{(0)},{ }_{1} \mathbf{u}$ and ${ }_{1} \tilde{\mathbf{u}}$, we obtain

$$
\begin{aligned}
\left(\hat{U}_{s}^{(\lambda)}\right)_{3}= & \frac{\beta \gamma}{315(2+3 \kappa)^{2}(4+\kappa)(1+\kappa)^{2}}\left\{\left[\left(2560+10932 \kappa+23252 \kappa^{2}+24606 \kappa^{3}+10995 \kappa^{4}\right.\right.\right. \\
& \left.\left.+1575 \kappa^{5}\right)+\epsilon_{1}\left(5920+27588 \kappa+63341 \kappa^{2}+70626 \kappa^{3}+32940 \kappa^{4}+4725 \kappa^{5}\right)\right] \\
& \left.+\eta\left[\left(2186+2807 \kappa+237 \kappa^{2}\right)+\tilde{\epsilon}_{1}\left(6530+10598 \kappa+3567 \kappa^{2}+315 \kappa^{3}\right)\right]\right\} \\
= & -\beta \gamma\left[M\left(\epsilon_{1}, \kappa\right)+\eta \tilde{M}\left(\tilde{\epsilon}_{1}, \kappa\right)\right] .
\end{aligned}
$$



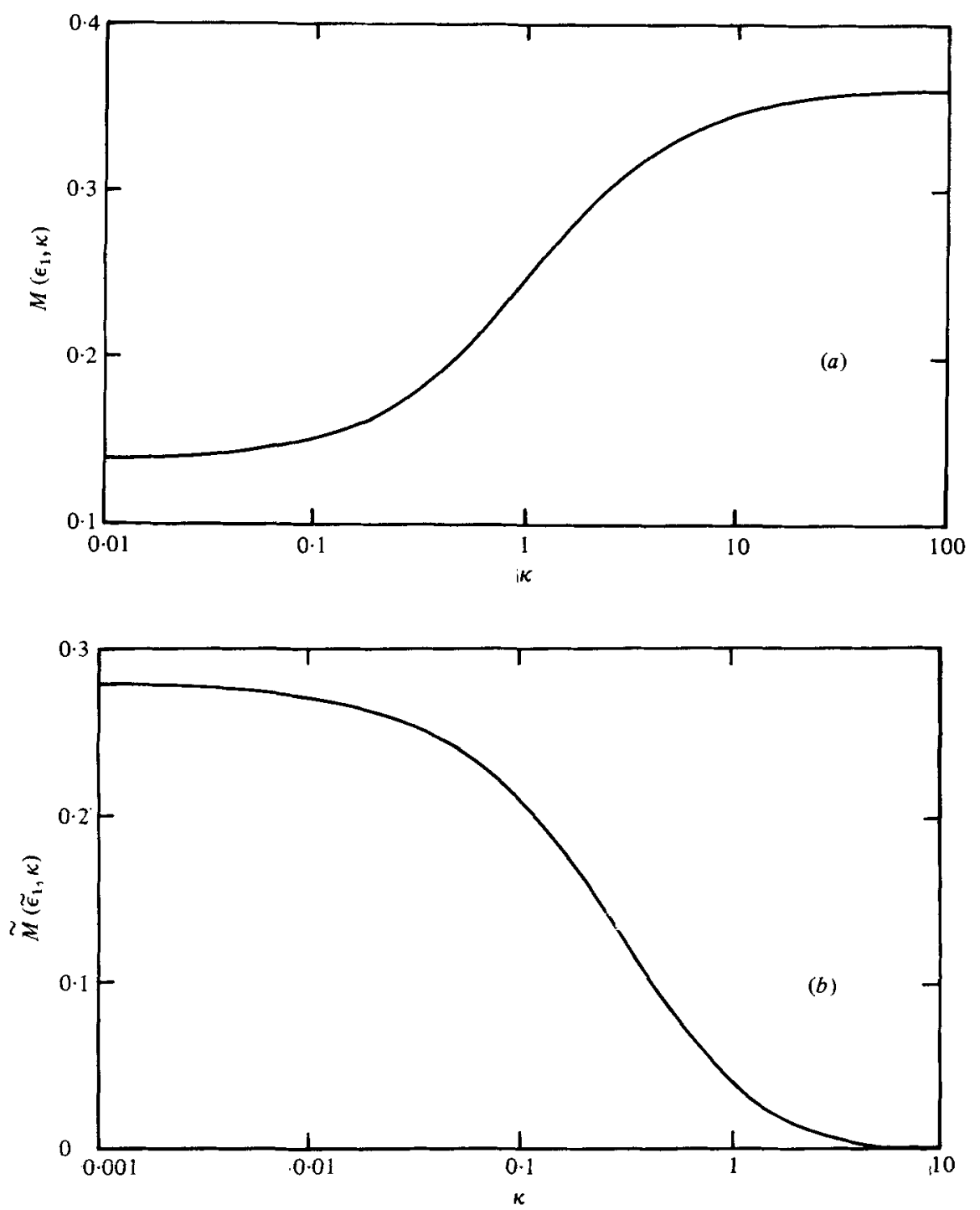

Figure 3. Migration velocity in a two-dimensional quadratic shearing flow due to: $(a)$ a nonNewtonian suspending fluid, $\epsilon_{1}=-0.55 ;(b)$ a non-Newtonian drop, $\tilde{\epsilon}_{1}=-0.55$.

We may note immediately that the migration velocity (5.8) is proportional to $\gamma$, and is thus identically zero in a linear shear flow. In this latter case, as we shall see shortly, the contributions to $\left(\hat{U}_{s}^{(\lambda)}\right)_{3}$ from the inner and outer regions, $V_{1}$ and $V_{2}$, are comparable in magnitude and it is necessary to explicitly consider wall reflexions. The parameter $\eta$ which appears in (5.8) represents a ratio of normal stress coefficients for the two fluids, i.e.

$$
\eta \equiv(\tilde{\lambda} / \lambda) \kappa=\phi_{3} / \phi_{3}
$$

and is thus independent of $\kappa$. For moderate values of $\kappa$, both fluids thus contribute to the migration velocity if $\eta$ is of $O(1)$. If $\eta$ approaches zero or infinity, one of the fluids may be considered Newtonian and therefore produces no direct contribution to $\left(\hat{U}_{s}\right)_{3}$ at this order. 
To determine the direction of migration, we must first estimate the material parameters $\epsilon_{1}$ and $\tilde{\epsilon}_{1}$ of the two fluids. In general, it is believed that their values should always lie between $-0 \cdot 5$ and $-0 \cdot 6$. The most significant experimental verification was obtained by Beavers \& Joseph (1975) for the motor oil additive STP. Using the cone and plate device they estimated $\phi_{2}$ to be $-2.78 \mathrm{~g} \mathrm{~cm}^{-1}$. In addition, the parameter $3 \phi_{2}+2 \phi_{3}$ was measured in rod climbing experiments as approximately $0.95 \mathrm{~g}$ $\mathrm{cm}^{-1}$. Thus $\epsilon_{1} \sim-0 \cdot 60$. This value agrees exactly with that estimated by Leal (1975) for a $3 \%$ PAA solution, based upon orbit drift experiments. For the above range of $\epsilon_{1}$ and $\tilde{\epsilon}_{1}$, it is easily seen that $M$ and $\tilde{M}$ are always positive, and hence migration is predicted to occur toward the region of smallest (absolute) shear rate, in agreement with the result of Ho \& Leal (1976) for the case of a rigid sphere.

In figure 3 we plot the functions $M$ and $\widetilde{M}$ which represent the separate contributions from the two fluids to (5.8), as functions of $\kappa$. When $\kappa$ approaches infinity, we see that the 'very viscous' drop has no direct effect on $\left(\hat{U}_{s}^{(\lambda)}\right)_{3}$, whereas the contribution of the suspending phase reduces to that calculated by Ho \& Leal (1976) for the rigid sphere problem. This is, of course, to be expected since the internal motion of the drop becomes that of a rotating rigid sphere for large $\kappa$. For decreasing values of $\kappa$, on the other hand, the contribution of the suspending fluid decreases whereas that of the drop fluid increases, until they reach limiting, non-zero values when $\kappa$ approaches zero. Obviously, this limit means that the drop has a much lower viscosity than the suspending fluid, but it also requires comparable values for the parameters $\phi_{3}$ and $\tilde{\phi}_{3}$. Thus, the limit $\kappa \rightarrow 0$ does not correspond to a gas bubble as might at first be supposed, and there is no paradox in a non-zero value for $\tilde{M}$ at $\kappa=0$.

\subsection{The linear unidirectional shear flow}

Let us now turn to the case of a linear unidirectional shear flow, for which the shear gradient $\gamma$ is zero. Here again, the calculations for the contributions of the suspending and drop fluids follow the procedures outlined previously. By dividing the entire volume $V_{f}$ into 'near-field' region $V_{1}$ and 'far-field' region $V_{2}$, we obtain the leading terms in $\zeta$ in the expression for the migration velocity. For $V_{1}$, the only contribution is of $O\left(\zeta^{4}\right)$ and arises from the interaction of the $O\left(\zeta^{2}\right)$ terms in $\left.{ }_{2} \mathbf{u},{ }_{3} \mathbf{u}\right)$ and the $O(\zeta)$ terms of ${ }_{1} \mathrm{U}^{(0)}$. For $V_{2}$, the leading contributions are also of $O\left(\zeta^{4}\right)$ and may arise in principle from any combinations between $\left({ }_{1} \mathbf{u},{ }_{2} \mathrm{u}\right)$ and $\left({ }_{1} \mathrm{U}^{(0)},{ }_{2} \mathrm{U}^{(0)}\right)$, expressed in outer variables. However, most of the terms in $V_{2}$ cancel each other after integration, with the remainder coming only from ${ }_{1} \mathbf{u}$ and ${ }_{1} U^{(0)}$. Unlike the $\beta \gamma$ contribution to the migration velocity which was shown in the previous subsection to be a 'near-field' effect, the contribution to the migration velocity in a linear shear flow is a result of hydrodynamic interaction between the particle and the walls. It is therefore not surprising that the integral over $V_{f}$ should contain contributions both from $V_{1}$ and $V_{2}$.

Inside the drop, the calculations are straightforward with the dominant terms coming from ${ }_{2+3} \tilde{\mathbf{u}}$ and ${ }_{1} \tilde{\mathbf{U}}^{(0)}$. When the contributions from both $V_{f}$ and $\tilde{V}_{f}$ are substituted into (5.7), we finally obtain an expression for the migration velocity (cf. Chan 1979)

$$
\begin{aligned}
.\left(\hat{U}_{s}^{(\lambda)}\right)_{3}= & -\frac{\zeta \beta^{2} J_{2}}{420(2+3 \kappa)(1+\kappa)^{2}}\left\{\left[\left(232+666 \kappa+1068 \kappa^{2}+455 \kappa^{3}\right)\right.\right. \\
& \left.\left.+\epsilon_{1}\left(928+3168 \kappa+4614 \kappa^{2}+2185 \kappa^{3}\right)\right]+12 \eta\left[31+\tilde{\epsilon}_{1}(108+105 \kappa)\right]\right\} .
\end{aligned}
$$



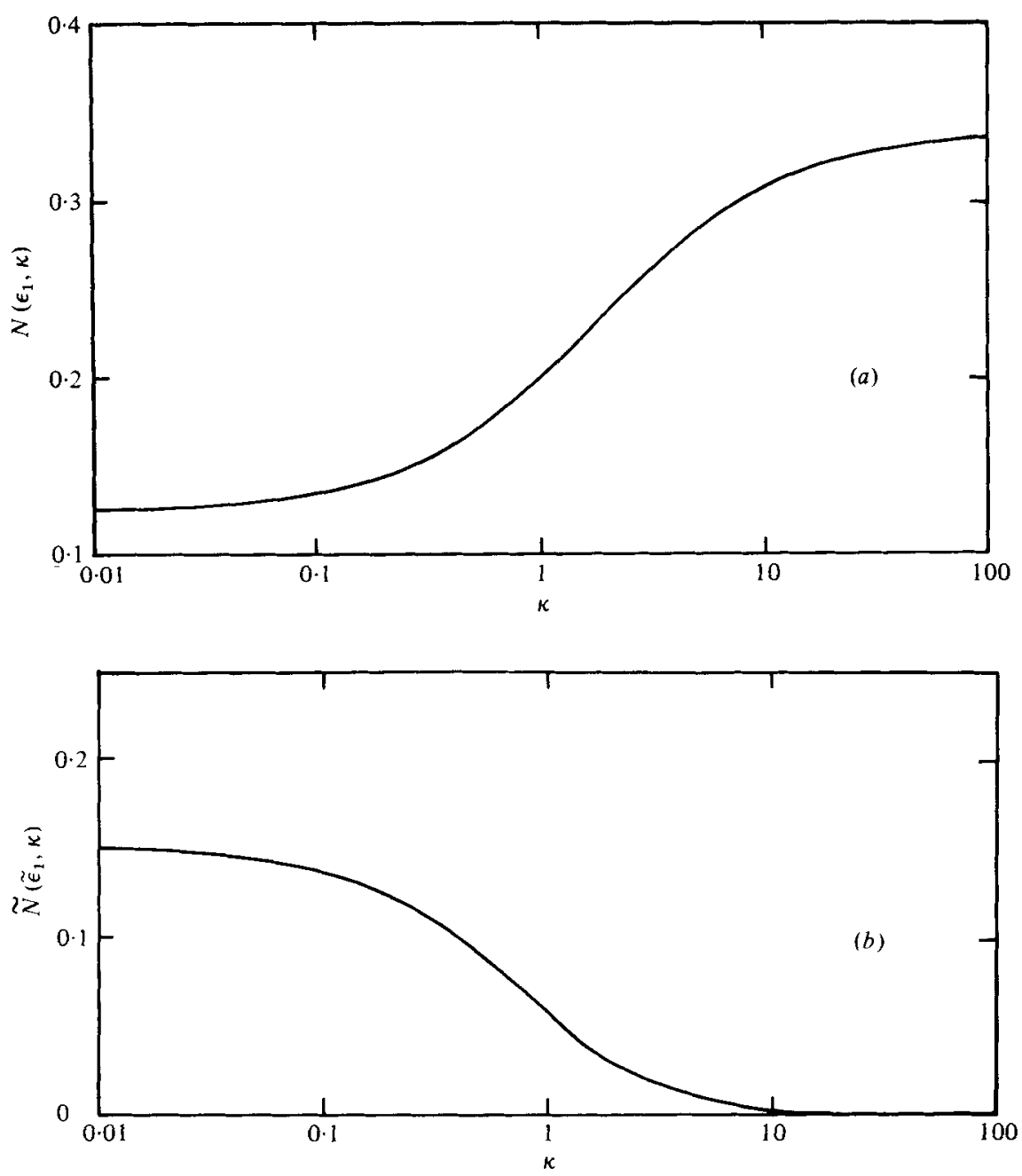

Figure 4. Migration velocity in a simple shear flow due to: (a) a non-Newtonian suspending fluid, $\epsilon_{1}=-\mathbf{0 . 5 5} ;(b)$ a non-Newtonian drop, $\widetilde{\epsilon}_{1}=-0.55$.

Using (4.23), this becomes

$$
\begin{aligned}
\left(\hat{U}_{s}^{(\lambda)}\right)_{3}= & -\frac{\zeta^{2} \beta^{2}}{2240(1+\kappa)^{3}}\left\{\left[\left(232+666 \kappa+1068 \kappa^{2}+445 \kappa^{3}\right)+\epsilon_{1}(928+3168 \kappa\right.\right. \\
& \left.\left.\left.+4614 \kappa^{2}+2185 \kappa^{3}\right)\right]+12 \eta\left[31+\tilde{\epsilon}_{1}(108+105 \kappa)\right]\right\}\left[\frac{1}{s^{2}}-\frac{1}{(1-s)^{2}}+2(1-2 s)\right] \\
= & \zeta^{2} \beta^{2}\left[N\left(\epsilon_{1}, \kappa\right)+\eta \tilde{N}\left(\tilde{\epsilon}_{1}, \kappa\right)\right]\left[\frac{1}{s^{2}}-\frac{1}{(1-s)^{2}}+2(1-2 s)\right]
\end{aligned}
$$

Once again, the functions $N\left(\epsilon_{1}, \kappa\right)$ and $\hat{N}\left(\tilde{\epsilon}_{1}, \kappa\right)$ are always positive for reasonable values of $\epsilon_{1}$ and $\tilde{\epsilon}_{1}$. Therefore the drop is predicted to migrate from the walls toward the centre-line. In figures $4(a)$ and $(b)$ we plot $N\left(\epsilon_{1}, \kappa\right)$ and $\tilde{N}\left(\tilde{\epsilon}_{1}, \kappa\right)$ as functions of $\kappa$. The dependence of $\left(\hat{U}_{s}^{(\lambda)}\right)_{3}$ on $\kappa$ is obviously similar to that predicted for the quadratic shear flow case. 


\subsection{Generalization to three-dimensional quadratic shear flows}

Although the analysis above was restricted to a two-dimensional undisturbed shearing flow, it is sometimes possible to generalize the results for the migration velocity to three-dimensional undisturbed motions of the same type, without the need to repeat the fluid dynamical calculations, by using the procedures which we have outlined in Chan \& Leal (1977). The essential requirements for this type of extension is that the effect must be localized near the particle, so that hydrodynamic interactions between the particle and walls play no role. To put it another way, the dominant contribution to the integral over $V_{f}$ in (5.7) must come from the inner region $V_{1}$ so that we effectively have migration in an unbounded domain with the undisturbed velocity profile, $(4.3 a)$, at infinity. Thus, the expression (5.8) for migration in a quadratic profile may be generalized using the analysis of Chan \& Leal (1977), but the contribution (5.10) due to hydrodynamic interaction with the walls in a linear shear flow is specifically excluded.

Let us first consider the case of a non-Newtonian spherical drop in a three-dimensional Poiseuille flow of a second-order fluid. For this purpose, we consider a circular tube with radius $B_{0}$. The distance of the drop from the tube centre at any instant is denoted as $D$. The undisturbed flow at infinity is expressed as

$$
\mathbf{V}=\left[\alpha+\beta x_{3}+\gamma\left(x_{3}^{2}+x_{2}^{2}\right)\right] \mathbf{e}_{1}-\hat{\mathbf{U}}_{s},
$$

where $x_{3}$ is now in the radial direction and

$$
\alpha=V_{\max }\left(1-D^{2} / B_{0}^{2}\right), \quad \beta=-2 V_{\max } a D / B_{0}^{2}, \quad \gamma=-V_{\max } a^{2} / B_{0}^{2} .
$$

By substituting (5.11) into the migration velocity expression of Chan \& Leal (1977), we thus obtain the result

$$
\begin{aligned}
\left(\hat{U}_{s}^{(\lambda)}\right)_{3}= & \frac{\beta \gamma}{630(2+3 \kappa)^{2}(1+\kappa)^{2}}\left\{\left[\left(1520+5172 \kappa+10594 \kappa^{2}+10560 \kappa^{3}+3465 \kappa^{4}\right)\right.\right. \\
& \left.+\epsilon_{1}\left(3200+12048 \kappa+27334 \kappa^{2}+28620 \kappa^{3}+9765 \kappa^{4}\right)\right] \\
& \left.+12 \eta\left[(158+174 \kappa)+\tilde{\epsilon}_{1}\left(425+564 \kappa+105 \kappa^{2}\right)\right]\right\} .
\end{aligned}
$$

This expression should be compared with (5.8). For the same values of $\beta$ and $\gamma$, we easily see that the qualitative behaviour of the two equations is very similar. Indeed, detailed numerical comparison shows that the difference in magnitude is never more than $30 \%$. The closest agreement occurs when $\kappa \rightarrow \infty$, in which case the discrepancy is only $10 \%$. In $\$ 8$, we shall compare (5.13) with the experimental data of Karnis \& Mason (1966) and of Gauthier et al. $(1971 a, b)$.

A second problem of considerable interest is the generalization of (5.8) to a Couette flow. It may be supposed that this could be approximated as a linear, unidirectional flow to which (5.10) is directly relevant. However, a small shear gradient always exists in the Couette device due to curvature and it is thus prudent, for values of $\zeta$ which are not vanishingly small, to consider both shear gradients and hydrodynamic interactions between the particle and walls in any comparison of the present theory with experimental observations. An attempt to apply (5.8) to examine the shear gradient effect in Couette flow was made by Ho \& Leal (1976) for rigid spherical particles using a local (and incorrect) two-dimensional approximation to the undisturbed velocity field. We shall comment on the validity of this approach later in 
this section. However, let us first apply the rigorous approach of Chan \& Leal (1977) to the generalization of (5.8) for a Couette velocity field.

We follow initially the analysis of Brunn (1976) and consider the full problem of two concentric cylinders (with radii $R_{1}, R_{2} ; R_{2}>R_{1}$ ) rotating with angular velocities $\Omega_{1}, \Omega_{2}$, respectively. The undisturbed velocity of the flow at any material point $(R, \phi)$ measured from the centre of the Couette device is then given as

$$
\mathbf{V}^{*}=\left(A_{1} R+A_{2} / R\right) \mathbf{e}_{\phi},
$$

where $\quad A_{1}=\left(\Omega_{2} R_{2}^{2}-\Omega_{1} R_{1}^{2}\right) /\left(R_{2}^{2}-R_{1}^{2}\right)$ and $A_{2}=R_{1}^{2} R_{2}^{2}\left(\Omega_{1}-\Omega_{2}\right) /\left(R_{2}^{2}-R_{1}^{2}\right)$.

We denote by $\left(x_{1}, x_{3}\right)$ the components of a position vector, non-dimensionalized with the drop radius $a$, which is defined relative to a co-ordinate system with origin at the drop centre, and the $x_{3}$ axis coincident with the radial unit vector of the natural cylindrical co-ordinates for the Couette device. The sphere centre is itself a distance $R_{0}$ from the axis of the Couette device. Thus,

$$
R^{2}=\left(R_{0}+a x_{3}\right)^{2}+a^{2} x_{1}^{2}
$$

Dividing (5.14) by the characteristic velocity $G a$, we may re-express it in dimensionless form as

$$
\mathbf{V}^{\prime}=\left[A_{1}^{\prime}+\frac{A_{2}^{\prime}}{\left(R_{0}+a x_{3}\right)^{2}+a^{2} x_{1}^{2}}\right]\left[\left(R_{0}+a x_{3}\right) \mathbf{e}_{1}-a x_{1} \mathbf{e}_{3}\right],
$$

where $A_{1}^{\prime}=A_{1} / G a$ and $A_{2}^{\prime}=A_{2} / G a$. Finally, by a Taylor's series expansion about $O$, we may write the undisturbed velocity $\mathbf{V}$ (relative to the drop) in the quadratic form

where

$$
\mathbf{V}=\alpha+\beta \cdot \mathbf{x}+\gamma: \mathbf{x} \mathbf{x}-\hat{\mathbf{U}}_{s}
$$

$$
\left.\begin{array}{l}
\alpha=\left.V^{\prime}\right|_{0}=\left(A_{1}^{\prime} R_{0}+A_{2}^{\prime} / R_{0}\right) \mathbf{e}_{1}, \\
\beta=\left.\nabla V^{\prime}\right|_{0}=A_{1}^{\prime} a\left(\mathbf{e}_{1} \mathbf{e}_{3}-\mathbf{e}_{3} \mathbf{e}_{1}\right)-\frac{A_{2}^{\prime} a}{R_{0}^{2}}\left(\mathbf{e}_{1} \mathbf{e}_{3}+\mathbf{e}_{3} \mathbf{e}_{1}\right), \\
\gamma=\left.\frac{1}{2} \nabla \nabla V^{\prime}\right|_{0}=\left[\frac{A_{2}^{\prime} a^{2}}{R_{0}^{3}}\right]\left(-\mathbf{e}_{1} \mathbf{e}_{1} \mathbf{e}_{1}+\mathbf{e}_{1} \mathbf{e}_{3} \mathbf{e}_{3}+\mathbf{e}_{3} \mathbf{e}_{3} \mathbf{e}_{1}+\mathbf{e}_{3} \mathbf{e}_{1} \mathbf{e}_{3}\right) .
\end{array}\right\}
$$

Substituting (5.19) into the migration velocity expression of Chan \& Leal (1977), $\uparrow$ we obtain

$$
\begin{aligned}
\left(\hat{U}_{s}^{(\lambda)}\right)_{3}= & -\frac{1}{63(1+\kappa)^{2}(2+3 \kappa)}\left\{\left[\left(256+816 \kappa+1238 \kappa^{2}+525 \kappa^{3}\right)\right.\right. \\
& \left.\left.+\epsilon_{1}\left(592+2112 \kappa+3254 \kappa^{2}+1365 \kappa^{3}\right)\right]+24 \eta\left(8+17 \tilde{\epsilon}_{1}\right)\right\}\left[\frac{A_{2}^{\prime 2} a^{3}}{R_{0}^{5}}\right] .
\end{aligned}
$$

In the limit of a rigid sphere, this becomes

$$
\left(\hat{U}_{s}^{(\lambda)}\right)_{3}=-\frac{5}{9}\left(5+13 \epsilon_{1}\right)\left(\frac{A_{2}^{\prime 2} a^{3}}{R_{0}^{5}}\right)
$$

which agrees exactly with the result of Brunn (1976).

Ho \& Leal (1976) have also obtained an expression for the migration velocity of

$\dagger$ Ignoring temporarily the fact that Chan \& Leal (1977) assumed no rotation of the particle co-ordinates, whereas the system used in (5.18) is clearly rotating. We shall see shortly, however, that for a Couette flow there is no correction to the migration velocity due to rotation. 
a rigid sphere in a Couette flow of a second-order fluid. In that paper, however, the undisturbed flow was assumed to be locally two-dimensional, and hence represented by $(2.6 a)$ with the parameters given as (in our present notation)

$$
\alpha=A_{1}^{\prime} R_{0}+A_{2}^{\prime} / R_{0}, \quad \beta=A_{1}^{\prime} a-A_{2}^{\prime} a / R_{0}^{2}, \quad \gamma=A_{2}^{\prime} a^{2} / R_{0}^{3} .
$$

By this approximation, the $x_{3}$ component of $\mathbf{V}$ has been neglected. The migration velocity for a rigid sphere is then predicted as

$$
\left(\hat{U}_{s}^{(\lambda)}\right)_{3}=\frac{5}{9}\left(1+3 \epsilon_{1}\right)\left(A_{1}^{\prime}-A_{2}^{\prime} / R_{0}^{2}\right) A_{2}^{\prime} a^{3} / R_{0}^{3}
$$

This expression, unlike the correct result (5.21), suggests a slight difference in migration rate depending upon whether the inner or outer cylinder is rotating, and this was reported in Ho \& Leal (1976) as being in qualitative agreement with the observations of Karnis \& Mason (1966). However, the result is incorrect† and the apparent agreement was simply fortuitous. It may be noted, in spite of this, that the direction of migration is the same as predicted by (5.21). Furthermore, for a Couette apparatus with 'small' curvature (i.e. $R_{1} / R_{2} \sim 1$ ), the constant $A_{1}^{\prime} \sim A_{2}^{\prime} / R_{0}^{2}$ so that (5.23) differs from (5.21) simply by a numerical factor $2\left(1+3 \epsilon_{1}\right) /\left(5+13 \epsilon_{1}\right)$. For $\epsilon_{1} \sim-0 \cdot 6$, which is a generally agreed value, this factor equals $\sim 0.6$ and the two results differ in magnitude by about $40 \%$.

Let us now return to the effect of rotation of the co-ordinates associated with a Couette device [see footnote immediately preceding (5.20)]. The general theory of Chan \& Leal (1977) which is the basis of (5.20) has only been developed for circumstances in which the co-ordinates attached to the particle centre and parallel to the flow boundaries are non-rotating. This is appropriate, for example, for any unidirectional undisturbed flow. Generally speaking, however, these 'particle coordinates' may be expected to rotate as well as translate, and the rotation will generate non-zero contributions to the time derivatives in the Rivlin-Ericksen tensors of the secondorder fluid expansion [cf. (2.3)]. We have shown (cf. Chan 1979) that this will lead to non-zero contributions dependent upon the rate of rotation, $\boldsymbol{\Omega}_{f}$ in the general expression for the migration velocity of the particle. Since these terms have no counterpart in the detailed unidirectional flow calculations of the present paper, it is not possible to determine their coefficients by comparison, and the results such as (5.20) are therefore incomplete if $\boldsymbol{\Omega}_{f} \neq 0$. As we have noted, this situation must be considered in the present calculation of particle migration in Couette flow, where it is necessary to choose a co-ordinate system whose $x_{3}$ axis always points in the radial direction with respect to the Couette device. Fortunately, however, for the velocity field (5.18) and (5.19), it can be shown (Chan 1979) by general tensorial arguments that there will be no additional contributions to the migrationivelocity from the rotation of the particle co ordinates. We therefore conclude that (5.20) is valid for any Couette flow.

It is clear that the expression (5.10), representing migration in a unidirectional flow due to hydrodynamic interactions between the particle and walls, should also be modified for application to a Couette flow device. Unfortunately, no simple method exists to determine the appropriate modifications short of re-solving the complete problem with the Couette geometry and velocity field inserted from the beginning.

$\dagger$ We are indebted to Dr P. Brunn for this remark. 
Thus, if we are to compare theoretical results with available data for this case we presently have no choice but to use (5.10) and (5.20); the comparison between (5.21) and (5.23) suggests that this may be qualitatively, or even quantitatively, correct provided we restrict our attention to a 'narrow gap' device which is the case in the existing experiments of Mason and co-workers. Although the profile curvature term is $O\left(\zeta^{3}\right)$ whereas the wall interaction term is $O\left(\zeta^{4}\right)$, the curvature in the undisturbed Couette flow is itself small in the 'narrow gap' device and so the two contributions may have numerically comparable values in practice. Since wall interactions cause migration toward the centre line [cf. $(5,10)]$, whereas the velocity profile curvature causes migration toward the outer cylinder [cf. (5.20)], the drop in Couette flow is generally expected to attain an intermediate equilibrium position where the two contributions cancel each other. By comparing the two expressions for typical experimental conditions, we have found in fact that the wall interaction effect, corresponding to $(5.10)$, should nearly always be dominant. Thus, the equilibrium position should be quite near the centre-line. However, this conclusion is in disagreement with existing experimental observations for rigid spheres in a viscoelastic fluid [cf. figure 8 of Karnis \& Mason (1966)], which indicate migration toward the outer cylinder. This discrepancy with present theory may result from the application of (5.10) directly to the Couette flow problem. Another possibility is that the conditions for validity of the theory, e.g. $\zeta \ll 1, \lambda \ll 1$, etc. are simply not satisfied well enough in the experiments to allow a detailed correspondence between theory and experiment. In this regard, it should be noted that the $4 \%$ PAA in water solution used by Karnis \& Mason (1966) is strongly viscoelastic in the deformation-rate range of interest and is therefore not modelled well as an $n$th order fluid; for example, it exhibits, a strong shear-thinning of the apparent viscosity. It has been found in other related problems (cf. Leal 1975) that predicted non-Newtonian contributions to particle motion in an unbounded fluid domain may, nevertheless, exhibit qualitative or even quantitative agreement with experiments in a strong viscoelastic fluid, provided $\phi_{2}$ and $\phi_{3}$ are determined from normal stress data in the shear-rate range of interest rather than at zero shear-rate as is strictly required for the $n$th order fluid approximation. In the present case, this is also true provided the comparison is made between data and (5.21) alone, rather than (5.21) and (5.10). Perhaps the influence of the wall effects is relatively less in a strongly viscoelastic fluid than is suggested by the comparison between (5.21) and (5.10) which we indicated above. In our opinion, it would be of considerable interest in settling these questions to perform further experiments using a fluid with 'proven' second-order fluid behaviour in the shear-rate range of interest.

\subsection{The $O(\lambda)$ velocity and pressure fields}

For the analysis of $\S 7$, and for examining the mechanism of migration, we need also to consider the $O(\lambda)$ velocity, pressure and stress fields defined in (5.1). To this end, it will be sufficient to consider only the two-dimensional quadratic shear flow problem in an unbounded fluid, where wall reflexions are neglected. Furthermore, since a detailed calculation is extremely complicated, we shall only attempt to determine the forms of $\left(\mathbf{U}^{(\lambda)}, P^{(\lambda)}, \mathbf{S}^{(\lambda)}\right)$, with identical expressions for $\left(\tilde{\mathbf{U}}^{(\lambda)}, \tilde{P}^{(\lambda)}, \widetilde{\mathbf{S}}^{(\lambda)}\right)+\dagger$ By keeping

† The problem of the motion of a sphere in a linear shear flow of second-order fluid was considered in the Ph.D. thesis of Peery (1966). 
only the relevant $\beta^{2}$ and $\beta \gamma$ terms, we find

$$
\begin{aligned}
\mathbf{U}^{(\lambda)}= & -\left(\hat{U}_{s}^{(\lambda)}\right)_{3}\left[\mathbf{e}_{3}-\frac{2+3 \kappa}{4(1+\kappa)}\left(\frac{1}{r} \mathbf{e}_{3}+\frac{x_{3}}{r^{3}} \mathbf{x}\right)-\frac{\kappa}{4(1+\kappa)}\left(\frac{1}{r^{3}} \mathbf{e}_{3}-\frac{3 x_{3}}{r^{5}} \mathbf{x}\right)\right] \\
& +\left(\beta^{2} \Psi_{1}+\beta \gamma \theta_{1}\right) x_{1} \mathbf{e}_{1}+\left(\beta^{2} \Psi_{2}+\beta \gamma \theta_{2}\right) x_{3} \mathbf{e}_{3}+x_{1}^{2}\left(\beta^{2} \Psi_{3}+\beta \gamma \theta_{3}\right) x_{3} \mathbf{e}_{3} \\
& +\left(\beta^{2} \Psi_{4}+\beta \gamma \theta_{4}\right) \mathbf{x}+x_{1}^{2}\left(\beta^{2} \Psi_{5}+\beta \gamma \theta_{5}\right) \mathbf{x} \\
P^{(\lambda)}= & \left(\hat{U}_{s}^{(\lambda)}\right)_{3} \frac{2+3 \kappa}{2(1+\kappa)} \frac{x_{3}}{r^{3}}+\left(\beta^{2} \Psi_{6}+\beta \gamma \theta_{6}\right)+x_{1}^{2}\left(\beta^{2} \Psi_{7}+\beta \gamma \theta_{7}\right) .
\end{aligned}
$$

Obviously, the $\left(\hat{U}_{s}^{(\lambda)}\right)_{3}$ terms of the above equations arise from (5.3). $\Psi_{1} \ldots \Psi_{7}$ and $\theta_{1} \ldots \theta_{7}$ are complicated functions of $x_{3}$ and $r$, which may be obtained by first calculating the last term of (5.2) using the Newtonian velocity, and then solving (5.1). In this problem, the $\Psi$ 's are even functions of $x_{3}$ whereas the $\theta$ 's are odd.

We may now substitute (5.24) and (5.25) into (5.2) and obtain an expression for $\mathbf{S}^{(\lambda)}$. This is then dotted with the unit normal to give the stress vector acting at any point on the particle surface. In general, both $\mathbf{U}^{(\lambda)}$ and $\mathbf{S}^{(\lambda)}$. $\mathbf{n}$ must satisfy matching conditions $(5.4 a)-(5.4 c)$ on the drop surface. However, once again it is the form of the stress vector which is of greatest practical interest, and we easily see that this must be analogous to (5.24). Therefore,

$$
\begin{aligned}
\mathbf{S}^{(\lambda)} \cdot \mathbf{n}= & -\left(\hat{U}_{s}^{(\lambda)}\right)_{3}\left[\frac{3(2+3 \kappa)}{2(1+\kappa)} x_{3} \mathbf{e}_{r}+\frac{3 \kappa}{2(1+\kappa)}\left(\mathbf{e}_{3}-3 x_{3} \mathbf{e}_{r}\right)\right]+\left(\beta^{2} \Psi_{8}+\beta \gamma \theta_{8}\right) x_{1} \mathbf{e}_{1} \\
& +\left(\beta^{2 \Psi_{9}}+\beta \gamma \theta_{9}\right) x_{3} \mathbf{e}_{3}+x_{1}^{2}\left(\beta^{2} \Psi_{10}+\beta \gamma \theta_{10}\right) x_{3} \mathbf{e}_{3} \\
& +\left(\beta^{2} \Psi_{11}+\beta \gamma \theta_{11}\right) \mathbf{e}_{r}+x_{1}^{2}\left(\beta^{2} \Psi_{12}+\beta \gamma \theta_{12}\right) \mathbf{e}_{r} .
\end{aligned}
$$

Let us now examine the above equation in more detail. For example, the component of the surface stress vector in the $x_{1}$ direction is obviously odd in $x_{1}$, regardless of the exact values of the $\Psi$ 's and the $\theta$ 's. Therefore the $x_{1}$ component of the stress vector at any material point $\left(x_{1}, x_{2}, x_{3}\right)$ is always balanced by its equal but opposite $x_{1}$ component at $\left(-x_{1}, x_{2}, x_{3}\right)$. As a result, there can be no net non-Newtonian force in the $x_{1}$ direction acting on the drop at this order, and the streamwise translational velocity of the drop will be unchanged. Similarly, if we consider the $x_{3}$ component of (5.26), we easily see that the $\beta^{2}$ contribution is odd in $x_{3}$, and hence can have no net effect on the drop motion. However, the $\beta \gamma$ contribution is even in $x_{3}$ and therefore has the same sign at $\left(x_{1}, x_{2}, x_{3}\right)$ and at $\left(x_{1}, x_{2},-x_{3}\right)$. As a result, lateral migration will occur in the $x_{3}$ direction. As noted above, these conclusions are independent of the detailed form of the $\Psi$ 's and the $\theta$ 's. Of course, no definite result can be obtained from (5.26) concerning such questions as the predicted sign or magnitude of the migration velocity without these functions, and this is the purpose of the reciprocal theorem calculations.

We note that the above considerations are in fact consistent with the 'hoop' thrust arguments which were tentatively proposed by Ho \& Leal (1976) as providing the mechanistic explanation of lateral migration of a rigid particle in an unbounded viscoelastic fluid. In a unidirectional shearing flow of a second-order fluid (without suspended particles), the tension along a straight streamline will obviously not result in a net force on any material point. On the other hand, if a particle is present, the streamlines are deformed and the tension along a streamline can then be partially 
converted to a sideways 'hoop' thrust. However, for a linear shear flow, the streamlines are symmetric on all 'sides' of a spherical particle [as is obvious from (5.24)], and hence these 'hoop' thrusts can have no net effect. Only in the presence of a shear gradient will the streamlines be asymmetric in the lateral direction. Then, on the side with a higher undisturbed shear rate the tension along streamlines will be greater than along streamlines on the other side, and hence will have a larger net force. This means that migration will occur in the direction of decreasing shear rate.

Finally, we should remark that the assumption of a spherical drop will not be valid in general, since the normal stress condition of $(5.4 c)$ is not then satisfied. However, any deformation due to $\mathbf{U}^{(\lambda)}$ and $\widetilde{\mathbf{U}}^{(\lambda)}$ can only occur at $O(\lambda \delta)$. Once again, it is not possible to calculate $f^{(\lambda \delta)}$ in detail; instead, we shall simply express it as

$$
f^{(\lambda \delta)}=\left(\beta^{2} \Psi_{13}+\beta \gamma \theta_{13}\right)+x_{1}^{2}\left(\beta^{2} \Psi_{14}+\beta \gamma \theta_{14}\right),
$$

where the $\Psi$ 's and $\theta$ 's have the same properties as before.

\section{The $O(\delta)$ problem}

In this section, we consider the motion of a Newtonian deformable drop suspended in a shearing flow of a Newtonian fluid. This problem has been examined by several previous investigators. In particular, Chaffey et al. $(1965,1967)$ have considered a simple shear flow in which the drop is near a single plane wall; in this case, wall reflexions are needed for migration to occur. In contrast, Wohl \& Rubinow (1974) and Wohl (1976) considered migration in a Poiseuille flow, where the effect of the shear gradient is expected to be significant. On this basis, these authors assumed that it was sufficient to consider the drop in 'unbounded' Poiseuille flow in order to determine the migration velocity. This assumption is not justified rigorously in their analysis, but it is obviously correct since the calculated migration velocities are asymptotically larger than those obtained by Chaffey et al. $(1965,1967)$ for small $\zeta$. However, the solution of Chaffey et al. $(1965,1967)$ is still of importance for the particular case of a simple shear flow, since the migration predicted by Wohl (1976) and Wohl \& Rubinow (1974) will then reduce to zero.

We shall now reconsider the problem of drop migration in a Newtonian fluid due to flow-induced deformations of the drop shape. As noted in the introduction to this paper, the original Chaffey et al. $(1965,1967)$ analysis for a linear flow was limited to a fluid bounded by a single plane wall. In addition, we have noted that there are strong reasons to doubt the accuracy of the Wohl (1976) and Wohl \& Rubinow (1974) results. As in the previous section, the calculations to be presented here will utilize the reciprocal theorem approach, i.e. (3.10).

The equations of motion for the Newtonian suspending fluid are

$$
\nabla \cdot \mathbf{S}^{(\delta)}=0, \quad \nabla \cdot \mathbf{U}^{(\delta)}=0
$$

with boundary conditions at large distances from the drop

$$
\left.\begin{array}{ll}
\mathbf{U}^{(\delta)} \rightarrow-\hat{\mathbf{U}}_{\mathcal{S}}^{(\delta)} & \text { as } r \rightarrow \infty, \\
\mathbf{U}^{(\delta)}=-\hat{\mathbf{U}}_{\mathcal{s}}^{(\delta)} & \text { on the walls. }
\end{array}\right\}
$$


The equations for the drop fluid are, of course, analogous to (6.1). The matching conditions on $r=1$, are from $(2.10 a)-(2.10 c),(2.15)$ and $(2.16)$,

$$
\begin{gathered}
\mathbf{U}^{(\delta)}+f^{(\delta)} \frac{\partial}{\partial r} \mathbf{U}^{(0)}=\tilde{\mathbf{U}}^{(\delta)}+f^{(\delta)} \frac{\partial}{\partial r} \widetilde{\mathbf{U}}^{(0)} \\
{\left[\mathbf{U}^{(\delta)}+f^{(\delta)} \frac{\partial}{\partial r} \mathbf{U}^{(0)}\right] \cdot \mathbf{e}_{r}-\mathbf{U}^{(0)} \cdot \nabla f^{(\delta)}=\left[\tilde{\mathbf{U}}^{(\delta)}+f^{(\delta)} \frac{\partial}{\partial r} \tilde{\mathbf{U}}^{(0)}\right] \cdot \mathbf{e}_{r}-\tilde{\mathbf{U}}^{(0)} \cdot \nabla f^{(\delta)}=0,} \\
{\left[\mathbf{S}^{(\delta)}+f^{(\delta)} \frac{\partial}{\partial r} \mathbf{S}^{(0)}\right] \cdot \mathbf{e}_{r}-\mathbf{S}^{(0)} \cdot \nabla f^{(\delta)}=\kappa\left[\left(\tilde{\mathbf{S}}^{(\delta)}+f^{(\delta)} \frac{\partial}{\partial r} \widetilde{\mathbf{S}}^{(0)}\right) \cdot \mathbf{e}_{r}-\tilde{\mathbf{S}}^{(0)} \cdot \nabla f^{(\delta)}\right]} \\
+\left[2 f^{(\delta)}+\nabla^{2} f^{(\delta)}\right] \nabla f^{(\delta)}-\left[2 f^{(\delta \delta)}+\nabla^{2} f^{(\delta \delta)}-2 f^{(\delta)} f^{(\delta)}\right] \mathbf{e}_{r} .
\end{gathered}
$$

Here, as in the case of non-Newtonian migration, we only need to consider (3.10) to obtain $\hat{\mathbf{U}}_{s}^{(\delta)}$. The $O(\delta)$ expression from $(3.10)$ is.

$$
\begin{aligned}
-\int_{\boldsymbol{A}_{d}}\left[\left(\mathbf{S}^{(\delta)}-\kappa \widetilde{\mathbf{S}}^{(\delta)}\right) \cdot \mathbf{u}-(\mathbf{t}-\kappa \tilde{\mathbf{t}}) \cdot \mathbf{U}^{(\delta)}-\kappa \tilde{\mathbf{t}} \cdot\left(\mathbf{U}^{(\delta)}-\tilde{\mathbf{U}}(\delta)\right)\right] \cdot \mathbf{e}_{r} d A \\
\\
-\frac{2 \pi(2+3 \kappa)}{1+\kappa}\left(1+J_{1}+J_{\mathbf{4}}\right)\left(\hat{U}_{s}^{(\delta)}\right)_{3}=0 .
\end{aligned}
$$

The integrands may be simplified using the appropriate matching conditions from $(3.4 a)-(3.4 c)$ and $(6.3 a)-(6.3 c)$. These give

$$
\begin{aligned}
& \left(\mathbf{S}^{(\delta)}-\kappa \tilde{\mathbf{S}}^{(\delta)}\right) \cdot \mathbf{u} \cdot \mathbf{e}_{r}=\left[-f^{(\delta)} \frac{\partial}{\partial r}\left(\mathbf{S}^{(0)}-\kappa \tilde{\mathbf{S}}^{(0)}\right) \cdot \mathbf{e}_{r}+\left(\mathbf{S}^{(0)}-\kappa \tilde{\mathbf{S}}^{(0)}\right) \cdot \nabla f^{(\delta)}\right. \\
& \left.+\nabla f^{(\delta)}\left(2 f^{(\delta)}+\nabla^{2} f^{(\delta)}\right)\right] \cdot \mathbf{u} \\
& -(\mathbf{t}-\kappa \tilde{\mathbf{t}}) \cdot \mathbf{U}^{(\delta)} \cdot \mathbf{e}_{r}=(\mathbf{t}-\kappa \tilde{\mathbf{t}}): \mathbf{e}_{r} \mathbf{e}_{r}\left[f^{(\delta)}\left(\frac{\partial}{\partial r} \mathbf{U}^{(0)}\right) \cdot \mathbf{e}_{r}-\mathbf{U}^{(0)} \cdot \nabla f^{(\delta)}\right], \\
& -\kappa \tilde{\mathbf{t}} \cdot\left(\mathbf{U}^{(\delta)}-\tilde{\mathbf{U}}^{(\delta)}\right) \cdot \mathbf{e}_{r}=\kappa \tilde{\mathbf{t}} \cdot \mathbf{e}_{r}\left[f^{(\delta)} \frac{\partial}{\partial r}\left(\mathbf{U}^{(0)}-\tilde{\mathbf{U}}^{(0)}\right)\right] .
\end{aligned}
$$

By rearranging, and neglecting the term $J_{1}+J_{4}$, we thus obtain

$$
\begin{aligned}
\left(\hat{U}_{s}^{(\delta)}\right)_{3}= & -\frac{1+\kappa}{2 \pi(2+3 \kappa)} \int_{A_{d}}\left\{\left[-f^{(\delta)} \frac{\partial}{\partial r}\left(\mathbf{S}^{(0)}-\kappa \tilde{\mathbf{S}}^{(0)}\right) \cdot \mathbf{e}_{r}+\left(\mathbf{S}^{(0)}-\kappa \tilde{\mathbf{S}}^{(0)}\right) \cdot \nabla f^{(\delta)}\right.\right. \\
& \left.+\nabla f^{(\delta)}\left(2 f^{(\delta)}+\nabla^{2} f^{(\delta)}\right)\right] \cdot \mathbf{u}+(\mathbf{t}-\kappa \tilde{\mathbf{t}}): \mathbf{e}_{r} \mathbf{e}_{r}\left[f^{(\delta)}\left(\frac{\partial}{\partial r} \mathbf{U}^{(0)}\right) \cdot \mathbf{e}_{r}-\mathbf{U}^{(0)} \cdot \nabla f^{(\delta)}\right] \\
& \left.+\kappa \tilde{\mathbf{t}} \cdot \mathbf{e}_{r} \cdot\left[f^{(\delta)} \frac{\partial}{\partial r}\left(\mathbf{U}^{(0)}-\tilde{\mathbf{U}}^{(0)}\right)\right]\right\} d A .
\end{aligned}
$$

We have now expressed the migration velocity at $O(\delta)$ in terms of integrals which involve only the Newtonian velocity and stress fields. In contrast to (5.7), only surface integrals on $r=1$ are involved, and hence wall effects will arise only indirectly in the integrands. [In (5.7), the domain of integration itself is bounded by the walls.] Here we obtain the order of magnitudes of the integrands by using the estimates for $\left({ }_{1} \mathbf{u},{ }_{2} \mathbf{u},{ }_{3} \mathbf{u}\right)$ and $\left({ }_{1} U^{(0)}{ }_{2} U^{(0)}{ }_{3} U^{(0)}\right)$ provided by Ho \& Leal (1974), evaluated on the drop surface. The estimates for $\tilde{\mathbf{u}}$ and $\widetilde{\mathbf{U}}^{(\mathbf{0})}$ are of course identical. We now calculate the dominant contributions for the two cases of quadratic and linear shear flows. 


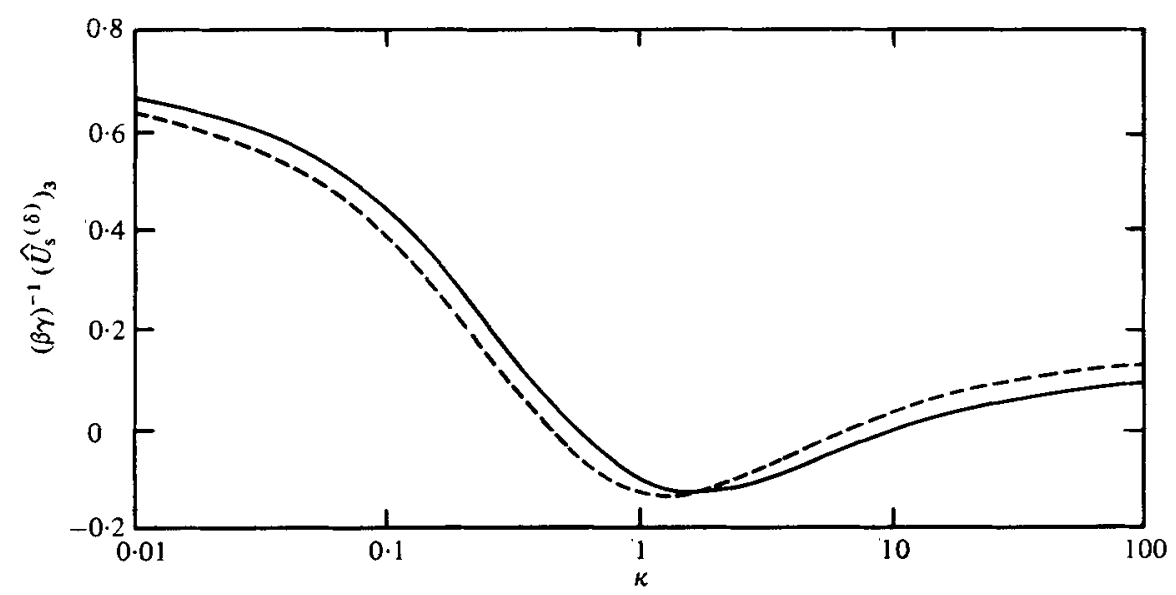

Figure 5. Migration velocity in a two-dimensional quadratic shearing flow due to drop deformation. - _ , present theory (6.8); - - , Wohl (1976).

\subsection{The quadratic unidirectional shear flow}

For a two-dimensional quadratic unidirectional shear flow, we can express $(6.6)$ in the form

$$
\hat{U}_{s}^{(\delta)} \sim \int_{A_{d}}\left[O\left(\zeta^{2}\right)+O\left(\zeta^{3}\right)+O\left(\zeta^{4}\right)\right] d A .
$$

Here, it can be shown easily that the $O\left(\zeta^{2}\right)$ term, which corresponds to the $\beta^{2}$ contribution, is odd in $x_{3}$ and hence integrates to zero. For the $O\left(\zeta^{3}\right)$ term, the only combination which is even in $x_{3}$ is proportional to $\beta \gamma$, and is obtained using ${ }_{1} \mathbf{u},{ }_{1} \tilde{\mathbf{u}},{ }_{1} U^{(0)}$, ${ }_{1} \widetilde{U}^{(0)}$ and ${ }_{1} f^{(\delta)}$. These terms correspond of course to the unbounded domain problem; thus Wohl's (1976) assumption that wall effects could be neglected at this order in $\zeta$ is justified. However, upon carrying out the detailed integrations for this dominant contribution, we obtain

$$
\begin{aligned}
\left(\hat{U}_{s}^{(\delta)}\right)_{3}= & -\frac{\beta \gamma}{(1+\kappa)^{2}(2+3 \kappa)}\left[\frac{16+19 \kappa}{42(2+3 \kappa)(4+\kappa)}\left(13-36 \kappa-73 \kappa^{2}-24 \kappa^{3}\right)\right. \\
& \left.+\frac{10+11 \kappa}{105}\left(8-\kappa+3 \kappa^{2}\right)\right] .
\end{aligned}
$$

This result is in disagreement with that of Wohl (1976), which is somewhat more complicated:

$$
\begin{aligned}
\left(\hat{U}_{s}^{(\delta)}\right)_{3}= & -\frac{\beta \gamma}{(1+\kappa)^{2}(2+3 \kappa)}\left[\frac { 1 6 + 1 9 \kappa } { 4 8 3 8 4 0 ( 2 + 3 \kappa ) ( 4 + \kappa ) } \left(138672-684612 \kappa-678210 \kappa^{2}\right.\right. \\
& \left.\left.-214362 \kappa^{3}+6237 \kappa^{4}\right)+\frac{10+11 \kappa}{940800}\left(71876-27898 \kappa+29691 \kappa^{2}\right)\right] .
\end{aligned}
$$

We are sceptical of the accuracy of the complicated expression that Wohl (1976) obtained, particularly in view of the fact that Wohl used the much more complicated 'direct' approach of calculating all variables at $O(\delta)$ in order to determine $\left(\hat{U}_{s}^{(\delta)}\right)_{3}$. However, on plotting the two migration velocities as functions of $\kappa$ (see figure 5), we find that the agreement between the theories is extremely good for $0.01 \leqslant \kappa \leqslant 100$. Interestingly, both theories predict that the direction of migration depends on the 
value of $\kappa$. For $\kappa$ between $\frac{1}{2}$ and 10 , the drop migrates toward the walls, while the inverse (toward the centre) is true for all other values of $\kappa$. Migration toward the walls has not previously been observed experimentally; however, so far as we are aware no experiments have yet been reported for $\frac{1}{2} \leqslant \kappa \leqslant 10$.

The expression (6.8) is, of course, limited to two-dimensional unidirectional flow. However, this dominant contribution to $\left(\hat{U}_{s}^{(\delta)}\right)_{3}$ corresponds to an unbounded domain, and it is thus possible to extend (6.8) to the important case of a three-dimensional Poiseuille flow. For this purpose, an expression for the $O(\delta)$ migration velocity of a deformable drop in a general quadratic flow of a Newtonian fluid is first needed. The coefficients in this expression are then determined by careful comparison with the two-dimensional problem. The required procedures are analogous to those of Chan \& Leal (1977), and will be discussed in detail in the appendix. For the undisturbed velocity profile, (5.11), of a three-dimensional Poiseuille flow, the final expression is

$$
\left(\hat{U}_{s}^{(\delta)}\right)_{3}=-\frac{\beta \gamma}{(1+\kappa)^{2}(2+3 \kappa)}\left[\frac{3}{14} \times \frac{16+19 \kappa}{2+3 \kappa}\left(1-\kappa-2 \kappa^{2}\right)+\frac{10+11 \kappa}{140}\left(8-\kappa+3 \kappa^{2}\right)\right] .
$$

Unfortunately, this disagrees quite significantly with the result of Wohl \& Rubinow (1974) for the same problem

$$
\begin{aligned}
\left(\hat{U}_{s}^{(\delta)}\right)_{3}= & -\frac{\beta \gamma}{(1+\kappa)^{2}(2+3 \kappa)}\left[\frac{16+19 \kappa}{26880(2+3 \kappa)}\left(27688+29354 \kappa+3741 \kappa^{2}-4284 \kappa^{3}\right)\right. \\
& \left.+\frac{10+11 \kappa}{78400}\left(14364-20191 \kappa+12310 \kappa^{2}\right)\right]
\end{aligned}
$$

Comparing (6.11) with (6.9), the magnitude of migration for three-dimensional Poiseuille flow is predicted by Wohl (1976) and Wohl \& Rubinow (1974) to be nearly ten times that for a two-dimensional Poiseuille flow when $\kappa$ approaches zero, provided that $\beta$ and $\gamma$ both remain the same. This prediction is at odds with intuition, according to which the qualitative behaviour of the two cases should be very similar. In fact, when we examine our own expressions [i.e. (6.8) and (6.10)], we find that they never differ by more than $50 \%$. We are confident that our calculations are correct. In $\$ 8$, we shall compare $(6.10)$ with previous experimental results.

\subsection{The linear unidirectional shear flow}

Let us now consider the case of simple (linear) shear flow, where the shear gradient $\gamma$ is zero. All $O\left(\zeta^{3}\right)$ terms of (6.7) will then vanish, and the leading contribution to the migration velocity is of $O\left(\zeta^{4}\right)$. We have found by careful consideration of the Newtonian velocity fields that the only relevant terms are $\left({ }_{2} \mathbf{u},{ }_{3} \mathbf{u}\right)$ and ${ }_{1} U^{(0)}$ from the suspending fluid, and ${ }_{2+3} \tilde{\mathbf{u}}$ and ${ }_{1} \widetilde{U}^{(0)}$ from the drop. By substitution into (6.6), we obtain

$$
\left(\hat{U}_{s}^{(\delta)}\right)_{3}=\zeta \beta^{2} J_{2} \frac{(16+19 \kappa)\left(54+97 \kappa+54 \kappa^{2}\right)}{280(1+\kappa)^{2}(2+3 \kappa)}
$$

which is then simplified using (4.23) to give

$$
\left(\hat{U}_{s}^{(\delta)}\right)_{3}=\zeta^{2} \beta^{2} \frac{3(16+19 \kappa)\left(54+97 \kappa+54 \kappa^{2}\right)}{4480(1+\kappa)^{3}}\left[\frac{1}{s^{2}}-\frac{1}{(1-s)^{2}}+2(1-2 s)\right] .
$$


By comparison, the migration velocity given by Chaffey et al. $(1965,1967)$ is

$$
\left(\hat{U}_{s}^{(\delta)}\right)_{3}=33 \zeta^{2} \beta^{2}(16+19 \kappa)\left(54+102 \kappa+54 \kappa^{2}\right) / 4480(1+\kappa)^{3} s^{2} .
$$

Both theories predict migration toward the centre-line of the apparatus. Obviously, the difference in dependence on radial position $s$ arises because we have considered the presence of two walls. When the drop is indeed much closer to either wall (i.e. $s \sim 0$ or $s \sim 1$ ), the last term in (6.13) reduces to $1 / s^{2}$ (or $1 /(1-s)^{2}$ ) as expected. Furthermore, if (6.14) is extended in an ad hoc manner with $1 / s^{2}$ replaced by

$$
1 / s^{2}-1 /\left(1-s^{2}\right)
$$

the dependence on $s$ will be quite similar to that of our full, two-wall analysis. It may be recalled, however, that (4.23) is itself an approximation of the full equation for $J_{2}$.

Let us examine the remaining parts of (6.13) and (6.14) more carefully. The factors $54+97 \kappa+54 \kappa^{2}$ and $54+102 \kappa+54 \kappa^{2}$ are, of course, effectively the same, hence the dependence on the viscosity ratio $\kappa$ is identical. However, the migration velocity predicted by Chaffey et al. $(1965,1967)$ is still seen to be exactly 11 times greater than ours. To explain this discrepancy, we have performed some of the calculations outlined in their paper, $\dagger$ and have indeed found that the ' 33 ' should actually be ' 3 ' instead. With this correction the two expressions essentially agree with each other.

Once again, it is difficult, in practice, to satisfy the assumption of a simple linear shear flow, since a small curvature always exists in a Couette device. The contribution of this curvature to particle motion can be calculated by first writing the velocity field as (5.11), and then using the general method developed in the appendix. Thus, we obtain

$$
\left(\widehat{U}_{s}^{(\delta)}\right)_{3}=-\frac{2\left(4+61 \kappa+85 \kappa^{2}+25 \kappa^{3}\right)}{7(2+3 \kappa)(1+\kappa)^{2}} \frac{A_{2}^{2} a^{3}}{R_{0}^{\prime 5}} .
$$

The above expression predicts that the curvature effect always causes migration toward the inner cylinder. [Quite surprisingly, if we use only the local two-dimensional approximation of Ho \& Leal (1976) to represent the bulk undisturbed velocity, the predicted direction would be toward the outer cylinder.] Thus, when both wall reflexion and shear gradient contributions are included, the drop should be expected to migrate to an equilibrium position which is between the centre-line and the inner wall. In spite of the fact that $(6.15)$ is $O\left(\zeta^{3}\right)$, and therefore asymptotically dominant for $\zeta \rightarrow 0$ over $(6.13)$ which is $O\left(\zeta^{4}\right)$, the velocity profile curvature is itself small for a 'narrow gap' Couette device so that the two effects may be expected to be of comparable magnitude. However, under the conditions of existing Couette flow experiments, comparison of (6.13) and (6.15) suggests that the wall interaction contribution dominates numerically and hence that the equilibrium position should be quite near the centre-line. This conclusion agrees very well with the experimental observations of Karnis \& Mason (1967). We shall present a more detailed comparison of experiment and theory in $\S 8$.

To calculate the contribution of the quadratic terms in the next section, we also need to obtain expressions for the $O(\delta)$ velocity and pressure fields. This is accomplished by following the same procedures as in the previous $O(\lambda)$ case. As expected,

$\dagger$ The number 33 first appears in the expression of $\tilde{u}_{v}$ in $\$ 5$ of Chaffey et al. $(1965,1967)$, and is therefore independent of the values of $A_{-3}^{(2)}$ and $A_{-3}$ from previous sections. Hence we only need to start from their equation (17) if we wish to oheck the validity of this coefficient. 
the predicted forms will be exactly the same as (5.24) and (5.25). We shall not repeat the cumbersome equations here.

\section{Higher order corrections}

We have now obtained estimates for the separate normal stress and deformation contributions to the migration velocity of the drop. In the presence of a shear gradient, their magnitudes are of $O\left(\lambda \zeta^{3}\right)$ and $O\left(\delta \zeta^{3}\right)$ respectively, whereas for a simple shear flow, they are of $O\left(\lambda \zeta^{4}\right)$ and $O\left(\delta \zeta^{4}\right)$. An obvious question that arises, at this stage, is whether these two terms are necessarily dominant over the quadratic combinations in all situations. For example, it is still possible that the next non-Newtonian contribution might be of $O\left(\lambda^{2} \zeta^{3}\right)$, thereby becoming important in the simple shear case for some values of $\lambda$ and $\zeta$. In order to verify or refute these possibilities, it is necessary to carry our calculations to higher order terms, $O\left(\lambda^{2}\right), O(\lambda \delta)$ and $O\left(\delta^{2}\right)$. Of course, these terms are of some intrinsic interest on their own. For example, the $O\left(\lambda^{2}\right)$ term in an $n$th order fluid expansion represents the first dependence of the viscosity on the shear rate [the $O(\lambda)$ term includes normal stresses only]. Similarly, the $O(\lambda \delta)$ contribution is of some interest because it represents the first interaction of normal stress and deformation effects.

For the $O\left(\lambda^{2}\right)$ problem, the equations of motion for the suspending phase are

$$
\nabla \cdot \mathbf{S}^{(\lambda \lambda)}=0 \text { and } \nabla \cdot \mathbf{U}^{(\lambda \lambda)}=0 \text {, }
$$

where

$$
\begin{aligned}
\mathbf{S}^{(\lambda \lambda)}= & -P^{(\lambda \lambda)}+\mathbf{D}_{(1)}^{(\lambda \lambda)}+\left[\mathbf{D}_{(1)}^{(0)} \cdot \mathbf{D}_{(1)}^{(\lambda)}+\mathbf{D}_{(1)}^{(\lambda)} \cdot \mathbf{D}_{(1)}^{(0)}+\epsilon_{1} \mathbf{D}_{(2)}^{(\lambda)}\right] \\
& +\left[\epsilon_{\mathbf{2}}\left(\mathbf{D}_{(1)}^{(0)}: \mathbf{D}_{(1)}^{(0)}\right) \mathbf{D}_{(1)}^{(0)}+\epsilon_{3} \mathbf{D}_{(3)}^{(0)}+\epsilon_{4}\left(\mathbf{D}_{(1)}^{(0)} \cdot \mathbf{D}_{(2)}^{(0)}+\mathbf{D}_{(2)}^{(0)} \cdot \mathbf{D}_{(1)}^{(0)}\right)\right] .
\end{aligned}
$$

The equations for the drop fluid are similar to (7.1) and (7.2). On the surface of the drop, the boundary conditions are

$$
\left.\begin{array}{l}
\mathbf{U}^{(\lambda \lambda)}=\tilde{\mathbf{U}}(\lambda \lambda), \quad \mathbf{U}^{(\lambda \lambda)} \cdot \mathbf{e}_{r}=\tilde{\mathbf{U}}^{(\lambda \lambda)} \cdot \mathbf{e}_{r}=0, \\
\mathbf{S}^{(\lambda \lambda)} \cdot \mathbf{e}_{r}=\kappa \tilde{\mathbf{S}}^{(\lambda \lambda)} \cdot \mathbf{e}_{r}-\left[2 f^{(\lambda \lambda \delta)}+\nabla^{2} f^{(\lambda \lambda \delta)}\right] \mathbf{e}_{r} .
\end{array}\right\}
$$

As before, we obtain an expression for the $O\left(\lambda^{2}\right)$ migration velocity from the reciprocal theorem. Hence, using (3.10), we get

$$
\begin{aligned}
& -\int_{A_{d}}\left[\left(\mathbf{S}^{(\lambda \lambda)}-\kappa \widetilde{\mathbf{S}}^{(\lambda \lambda)}\right) \cdot \mathbf{u}-(\mathbf{t}-\kappa \tilde{\mathbf{t}}) \cdot \mathbf{U}^{(\lambda \lambda)}\right. \\
& \left.-\kappa \tilde{\mathbf{t}} \cdot\left(\mathbf{U}^{(\lambda \lambda)}-\tilde{\mathbf{U}}^{(\lambda \lambda)}\right)-\mathbf{T}^{(\lambda \lambda)} \cdot \mathbf{u}\right] \cdot \mathbf{e}_{r} d A-2 \pi \frac{2+3 \kappa}{1+\kappa}\left(\hat{U}_{s}^{(\lambda \lambda)}\right)_{3} \\
& =\int_{V_{f}}\left[\left(\mathbf{D}_{(1)}^{(0)} \cdot \mathbf{D}_{(1)}^{(\lambda)}+\mathbf{D}_{(1)}^{(\lambda)} \cdot \mathbf{D}_{(1)}^{(0)}-\mathbf{E}_{(1)}^{(0)} \cdot \mathbf{E}_{(1)}^{(\lambda)}-\mathbf{E}_{(1)}^{(\lambda)} \cdot \mathbf{E}_{(1)}^{(0)}\right)+\epsilon_{1}\left(\mathbf{D}_{(2)}^{(\lambda)}-\mathbf{E}_{(2)}^{(\lambda)}\right)\right]: \nabla \mathbf{u} d V \\
& \quad+\kappa \frac{\lambda}{\lambda} \int_{\tilde{V}_{f}}\left[\tilde{\mathbf{D}}_{(1)}^{(0)} \cdot \tilde{\mathbf{D}}_{(1)}^{(\lambda)}+\widetilde{\mathbf{D}}_{(1)}^{(\lambda)} \cdot \tilde{\mathbf{D}}_{(1)}^{(0)}+\tilde{\epsilon}_{1} \tilde{\mathbf{D}}_{(2)}^{(\lambda)}\right]: \nabla \mathbf{u} d V \\
& \quad+\int_{V_{f}}\left[\epsilon_{2}, \epsilon_{3}, \epsilon_{4} \text { terms }\right]^{(0)}: \nabla \mathbf{u} d V+\kappa\left(\frac{\tilde{\lambda}}{\lambda}\right)^{2} \int_{\tilde{V}_{f}}\left[\tilde{\epsilon}_{2}, \tilde{\epsilon}_{3}, \tilde{\epsilon}_{4} \text { terms }\right]^{(0)}: \nabla \tilde{\mathbf{u}} d V \cdot
\end{aligned}
$$

Once again, the integrand of the surface integral over $A_{d}$ is identically zero. Furthermore, using the Newtonian solutions in conjunction with (5.24) for the $O(\lambda)$ velocity field, we can easily show that the integrands of the volume integrals over $V_{f}$ and $\tilde{V}_{f}$ are all odd in $x_{1}$ and therefore integrate to zero. Hence there will be no migration at 
this order. This conclusion is reached without any assumptions about wall effects and shear gradients, and will therefore be true for all unidirectional shear flows. This means that, to this order, the shear-dependence of viscosity yields no contribution to the migration of a spherical drop. Parenthetically, we may note that a similar result may be proved rigorously for any purely-viscous fluid model; i.e. for any fluid in which the only non-Newtonian property is a shear-dependent viscosity. This latter conclusion follows trivially from the 'mirror-symmetry time-reversal' theorem of Bretherton (1962), which takes into account the invariant nature of the NavierStokes equations (cf. Chan 1979).

Finally, the $O(\lambda \delta)$ and $O\left(\delta^{2}\right)$ contributions can be considered in the same manner. By keeping only relevant terms from the governing equations of $\S 3$, we obtain expressions for these two cases that are analogous to (7.1)-(7.4). Again, the integrands are odd in $x_{1}$. Hence there will be no correction to the migration velocity at any of the orders, $O\left(\lambda^{2}\right), O(\lambda \delta)$ and $O\left(\delta^{2}\right)$.

\section{Discussion}

We now wish to compare our calculations from the last three sections with the available experimental results of Mason and his co-workers (Goldsmith \& Mason 1962; Karnis \& Mason 1966, 1967; Gauthier et al. 1971a,b) for the motion of a deformable drop in a non-Newtonian fluid undergoing Couette or three-dimensional Poiseuille flow. First, we consider the migration of a rigid sphere (i.e. $\kappa \rightarrow \infty$ ) due to normal stresses alone. For a Couette flow, we have noted earlier that the profile curvature in a typical Couette device is usually small and hence the asymptotically dominant profile curvature contribution, which is $O\left(\zeta^{3}\right)$, may actually be numerically dominated by the $O\left(\zeta^{4}\right)$ wall interaction effect. On this basis, our theory predicts an equilibrium position quite near the centre-line, in contrast to the observations of Karnis \& Mason (1966) for sphere migration in a $4 \%$ solution of PAA in water. It is, in fact, striking that the experimental observations seem to agree qualitatively with the predicted migration rate and direction from the profile curvature effect with no account taken of wall interactions. For example, by integrating (5.21) alone, we obtain in dimensional variables (defined in $\$ 5.3$ )

$$
R_{0}^{6}-R_{0}^{6}(0)=\left[-\frac{\phi_{3}}{\mu_{0}}\left(5+13 \epsilon_{1}\right)\right]\left[\frac{10}{3} A_{2}^{2} a^{2}\right] T,
$$

where $T$ is the elapsed time. This equation can be compared with the data in figure 8 of Karnis \& Mason (1966). In particular, we may obtain an estimate of the rheological constant $\phi_{3} / \mu_{0}$ for $4 \%$ PAA in water, simply by fitting their data with (8.1). Thus, in figure 6, we plot $R_{0}^{6}$ as a function of $T$. From the slopes of the best fit straight lines, we estimate values for the parameter $-\phi_{3} / \mu_{0}\left(5+13 \epsilon_{1}\right)$ of $22 \cdot 8,47 \cdot 3,18 \cdot 1$ and $98.7 \mathrm{~s}$. These are quite reasonable for $4 \%$ PAA in water [cf. the estimates by Leal (1975) for $3 \%$ PAA in water]. In addition, it should be noted that PAA in water is strongly viscoelastic, in contrast to the 'near Newtonian' second-order fluid behaviour on which the present theory is based. A reasonable inference is thus that non-Newtonian contributions to particle motion, due to profile curvature, are reasonably well "predicted' by the theory for an unbounded second-order fluid; on the other hand, it appears that the wall interaction effects, which are dominant in a second-order fluid, 


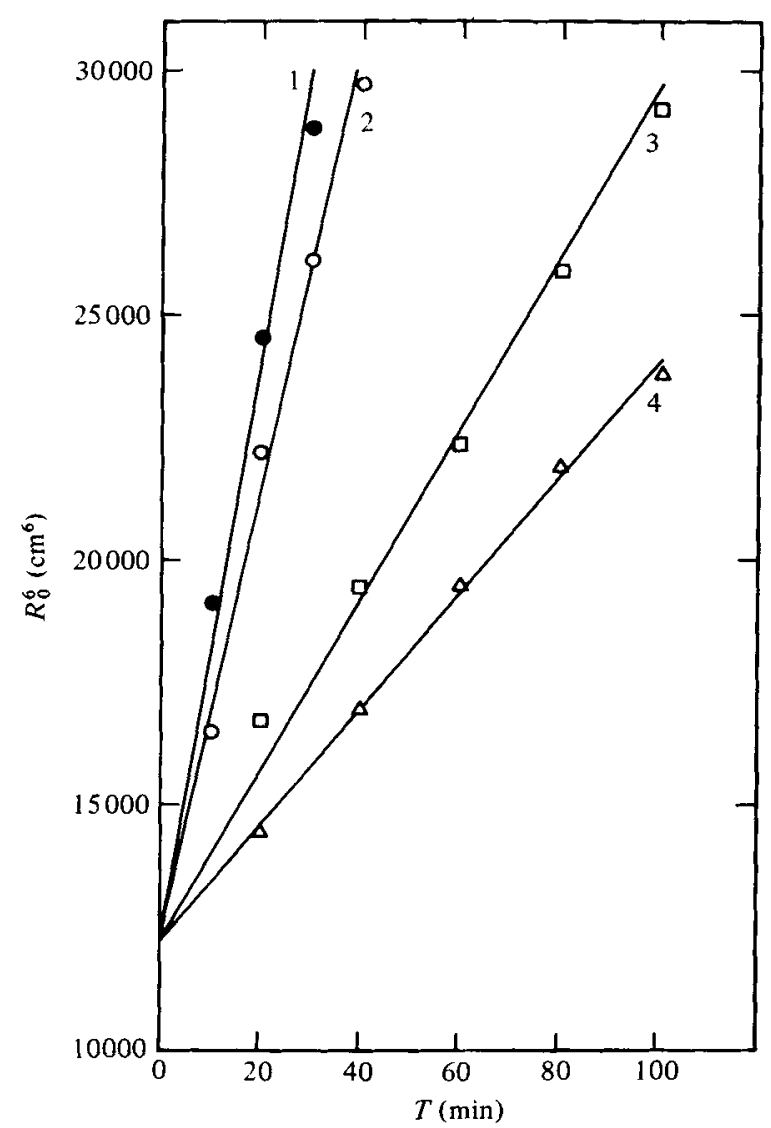

FigURe 6. $R_{0}^{8}$ vs. $T$ for a rigid sphere in a Couette flow $\left(R_{1}=4 \cdot 644 \mathrm{~cm}, K_{2}=5.795 \mathrm{~cm}\right)$ of $4 \%$ PAA solution in water, observed by Karnis \& Mason (1966). O, case 1: $a=0 \cdot 065 \mathrm{~cm}$, $\Omega_{1}=-0.092 \mathrm{rad} \mathrm{s} \mathrm{s}^{-1} ; \bigcirc$, case $2: a=0.065 \mathrm{~cm}, \Omega_{1}=0.0563 \mathrm{rad} \mathrm{sec}{ }^{-1} ; \square$, case $3: a=0.065 \mathrm{~cm}$, $\Omega_{2}=0.0563 \mathrm{rad} \mathrm{s}{ }^{-1} ; \triangle$, case $4: a=0.014 \mathrm{~cm}, \Omega_{1}=-0.092 \mathrm{rad} \mathrm{s}^{-1}$.

are greatly over-estimated when an attempt is made to extend the theory to the case of a strong viscoelastic liquid. Further experiments are presently being performed in our laboratory in an attempt to shed some light on these admittedly speculative ideas for the case of migration in a 'narrow' gap Couette device.

For a Poiseuille flow, in contrast to the narrow-gap Couette flow, the profile curvature is not 'small' and thus for small particles (i.e. $\zeta \ll 1$ ) the $O\left(\zeta^{3}\right)$ profile curvature contribution to the migration velocity is asymptotically and numerically dominant over the $O\left(\zeta^{4}\right)$ wall-particle hydrodynamic interaction effects. In the limit of $\kappa \rightarrow \infty$, (5.13) reduces to

$$
\left(\hat{U}_{s}^{(\lambda)}\right)_{3}=\frac{1}{18} \beta \gamma\left(11+31 \epsilon_{1}\right)
$$

By dividing this expression with the axial velocity and then integrating, we obtain an equation for the trajectory of the sphere. In dimensional variables, we have

$$
\begin{aligned}
{\left[\frac{D^{2}}{2 B_{0}^{2}}-\left(1-\frac{2}{3} \frac{a^{2}}{B_{0}^{2}}\right) \ln \frac{D}{B_{0}}\right]-\left[\frac{D(0)^{2}}{2 B_{0}^{2}}-\left(1-\frac{2}{3} \frac{a^{2}}{B_{0}^{2}}\right) \ln \frac{D(0)}{B_{0}}\right] } \\
=\left[-\frac{\phi_{3}}{\mu_{0}}\left(11+31 \epsilon_{1}\right)\right]\left[\frac{V_{\max }^{*} a^{2}}{9 B_{0}^{4}}\right][L-L(0)],
\end{aligned}
$$




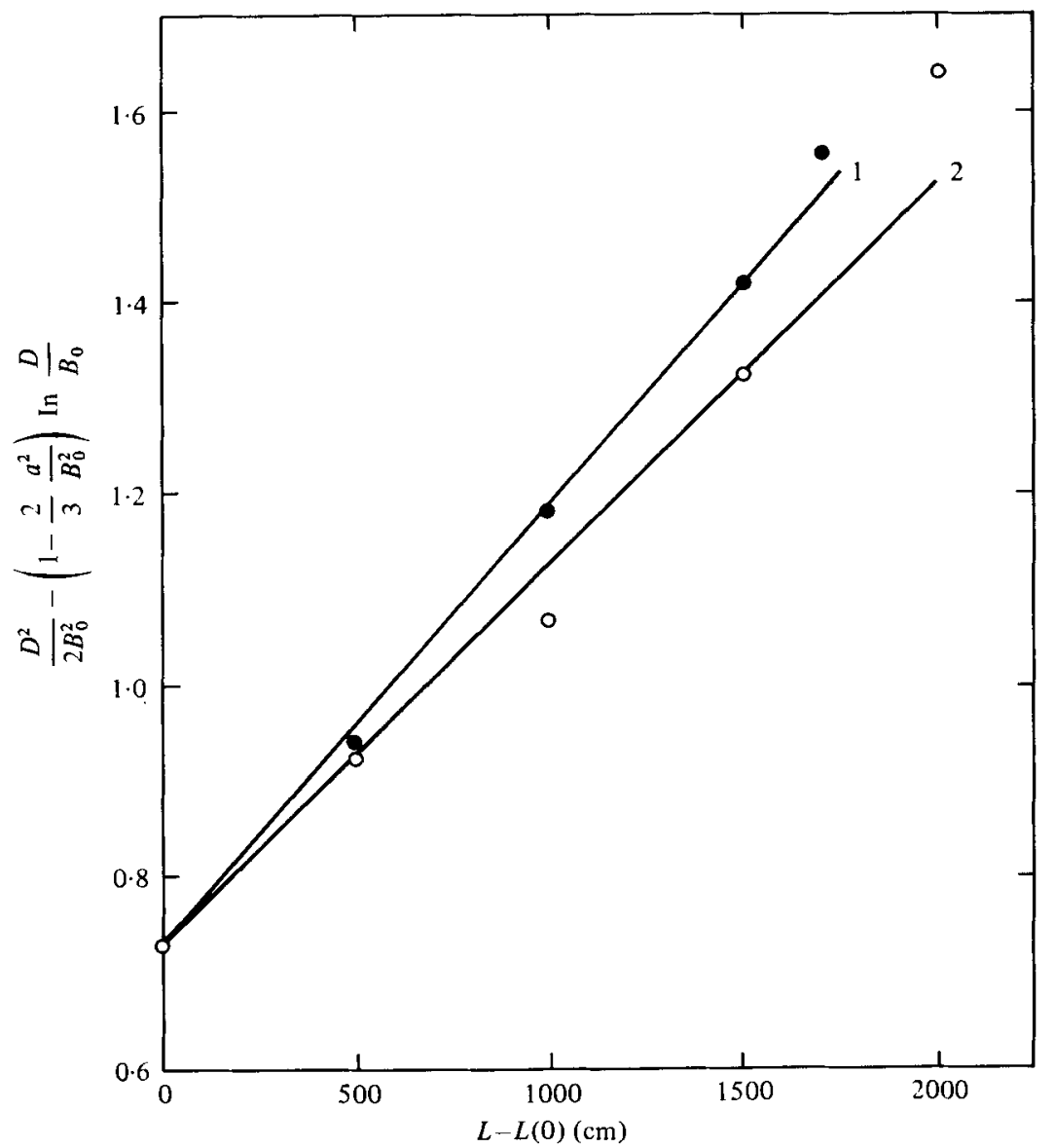

Figure 7. $D^{2} / 2 B_{0}^{2}-\left(1-\frac{2}{3} a^{2} / B_{0}^{2}\right) \ln \left(D / B_{0}\right)$ vs. $L-L(0)$ for a rigid sphere in a three-dimensional Poiseuille flow $\left(B_{0}=0.3 \mathrm{~cm}, V_{\max }^{*}=0.487 \mathrm{~cm} \mathrm{~s}^{-1}\right)$ of $6 \%$ PIB solution in Decalin, observed by Karnis \& Mason (1966). O, case $1: a / B_{0}=0.037 ; \bigcirc$, case $2: a / B_{0}=0.027$; - best linear fit with data.

where $L-L(0)$ is the axial distance travelled by the sphere; all other variables are again defined in $\$ 5.3$. In figure 7 , we plot $D^{2} / 2 B_{0}^{2}-\left(1-\frac{2}{3} a^{2} / B_{0}^{2}\right) \ln \left(D / B_{0}\right)$ as calculated from the data in figure 4 of Karnis \& Mason (1966) for sphere migration in a $6 \%$ solution of PIB in Decalin, as a function of $L-L(0)$ for particles with $a / B_{0}=0.037$ and 0.027 respectively. The parameter $-\phi_{3}\left(11+31 \epsilon_{1}\right) / \mu_{0}$ is estimated to be 0.91 and $0.56 \mathrm{~s}$. These values are certainly reasonable for $6 \%$ PIB in Decalin, but we have no direct rheological data for comparison. The difference between the two cases is believed by us to result from the change in particle size. It will be recalled that the present theory is strictly applicable only in the limit $a / B_{0} \rightarrow 0$. In particular, direct contributions to the migration rate due to hydrodynamic interaction between the particle and tube walls is not included in (8.2) - this effect will become more important for larger $a / B_{0}$ and thus lead to faster migration or increasing apparent values for $-\left(\phi_{3} / \mu_{0}\right)\left(11+31 \epsilon_{1}\right)$ as a function of $a / B_{0}$. In order to estimate the magnitude of this effect, one would have to extend the present theory to include wall reflexions for a quadratic velocity profile (recall, that we have so far included wall reflexions only 
for a linear profile, where they provide the only non-zero contribution to the migration rate). A second factor, also associated with the particle size, which may influence the apparent magnitude of $-\left(\phi_{3} / \mu_{0}\right)\left(11+31 \epsilon_{1}\right)$, is the fact that the local strain-rates (i.e. those associated with the particle-induced disturbance flow) will depend upon $a$. Obviously, it is very difficult to estimate the contribution of this effect. However, it seems to go in the same direction as the change which is observed, since $\phi_{3}$ is a decreasing function of shear-rate.

Let us now turn to the trajectory for a deformable Newtonian drop in a Newtonian suspending fluid. In this case, all of the rheological constants can be measured, and bence our predictions can be compared directly with known experimental observations. For a Couette flow, the migration rate again includes contributions from the interaction of the particle with the bounding walls [toward the centre-line, ef. (6.13)], and also from the shear gradient of a Couette device [toward the inner cylinder, cf. (6.15)]. Thus, the drop is expected to attain, in general, an equilibrium position between the centre-line and the inner cylinder, where the two effects cancel each other. For the experimental conditions of Karnis \& Mason (1967), the wall contributions always dominate numerically, and hence the predicted equilibrium position is quite near the centre-line, in agreement with their experimental observations. To obtain the predicted trajectories, we add the two contributions and then integrate to obtain

where

$$
T=\frac{d \sigma}{\mu_{0}} \int_{s_{0}}^{s} \frac{d s}{Z(s)}
$$

$$
\begin{aligned}
Z(s)= & \frac{a^{4}}{d^{2}}\left(A_{1}-\frac{A_{2}}{R_{0}^{2}}\right)^{2} \frac{3(16+19 \kappa)\left(54+97 \kappa+54 \kappa^{2}\right)}{4480(1+\kappa)^{3}}\left[\frac{1}{s^{2}}-\frac{1}{(1-s)^{2}}+2(1-2 s)\right] \\
& -\frac{2\left(4+61 \kappa+85 \kappa^{2}+25 \kappa^{3}\right)}{7(2+3 \kappa)(1+\kappa)^{2}} \frac{A_{2}^{2} a^{3}}{R_{0}^{5}} .
\end{aligned}
$$

We now evaluate (8.4) numerically and then plot the predicted trajectory of the drop in figure 8 for the various conditions of Karnis \& Mason's (1967) experiments, together with the measured trajectory data. For cases 1 and 2, the measured rates are larger than our calculations, but the inverse is true for case 3 . Furthermore, the predicted equilibrium positions of $s=0.474,0.484$ and 0.487 for cases 1,2 and 3 [obtained by solving $Z(s)=0$ ] agree well with the experimental observations (for example, the measured equilibrium position for case 3 is at approximately $s=0 \cdot 47$ ).

For the sake of comparison, we have also calculated the corresponding theoretical results from Chaffey et al. $(1965,1967)$, which include only the interaction of the particle with one wall. These may be obtained approximately by dividing $T$ by 11 for each value of $s$. As shown in case 1 (which offers their best fit with data), their agreement with experiment is obviously much poorer than for our present theory.

It should be remarked that the conditions of the experiments do not lend themselves well to a definitive comparison between experiment and theory. Aside from $\zeta=a / d \ll 1$, it is also inherently assumed in our theory that the drop is not close to either wall (i.e. $\zeta \ll s$ for $0<s \leqslant 0.5$; $\zeta \ll 1-s$ for $0.5<s<1$ ). Now, the experimental values of $\zeta$ were $0.0337,0.0556$ and 0.0772 for cases 1,2 and 3 respectively. In addition, the initial values of $s$ (or $1-s$ for $0.5<s$ ) were $0.0526,0.0877$ and 0.149 , which are twice 


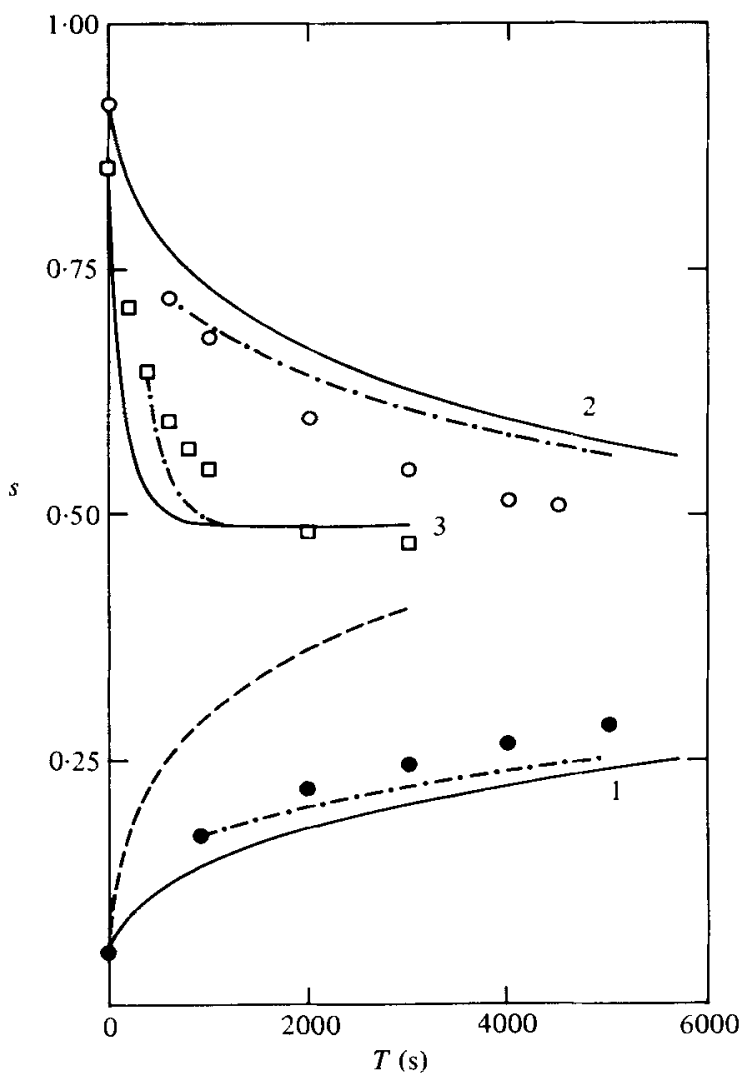

Figure 8. A comparison of experimental trajectory of a deformable drop in a simple shear flow with theory. From Karnis \& Mason (1967), $\kappa=2 \times 10^{-4}, \mu_{0}=50 \mathrm{P}, d=1.869 \mathrm{~cm}$, $\sigma=10 \mathrm{dyne} \mathrm{cm}^{-1}$. $O$, case $1: a=0.063 \mathrm{~cm}, V_{w}^{*}=0.662 \mathrm{~cm} \mathrm{~s}^{-1} ; 0$, case $2: a=0.104 \mathrm{~cm}$, $V_{w}^{*}=0.662 \mathrm{~cm} \mathrm{~s}^{-1} ; \square$, case $3: a=0.135 \mathrm{~cm}, V_{w}^{*}=1.835 \mathrm{~cm} \mathrm{~s}^{-1} ;-$, present theory $(8.3) ;$ $-\cdot-$, present theory but with comparison restricted to $5 \zeta<s$; ---, Chaffey et al. $(1965,1967)$ theory.

as large. The value of $\zeta$ is obviously fixed for a particular experiment. The maximum values of $s$ can, however, be varied to some degree by restricting our comparison only to those portions of the trajectories where the particle is 'near' to the centreline, say $5 \zeta \leqslant s$. Then, we see from figure 8 that the differences between theory and experiment are considerably reduced, with the best agreement occurring for the smallest drop used.

For a three-dimensional Poiseuille flow, we follow procedures analogous to those outlined by Wohl \& Rubinow (1974) to obtain

$$
\begin{gathered}
{\left[\frac{D^{2}}{2 B_{0}^{2}}-\left(1-\frac{2 \kappa}{2+3 \kappa} \frac{a^{2}}{B_{0}^{2}}\right) \ln \frac{D}{B_{0}}\right]-\left[\frac{D(0)^{2}}{2 B_{0}^{2}}-\left(1-\frac{2 \kappa}{2+3 \kappa} \frac{a^{2}}{B_{0}^{2}}\right) \ln \frac{D(0)}{B_{0}}\right]} \\
=\frac{2 V_{\max }^{*} a^{3} \mu_{0}}{B_{0}^{4} \sigma} \frac{1}{(1+\kappa)^{2}(2+3 \kappa)}\left[\frac{3}{14} \times \frac{16+19 \kappa}{2+3 \kappa}\left(1-\kappa-2 \kappa^{2}\right)\right. \\
\left.+\frac{10+11 \kappa}{140}\left(8-\kappa+3 \kappa^{2}\right)\right] \times[L-L(0)] .
\end{gathered}
$$

Here, we compare the above expression with the experimental observations of 


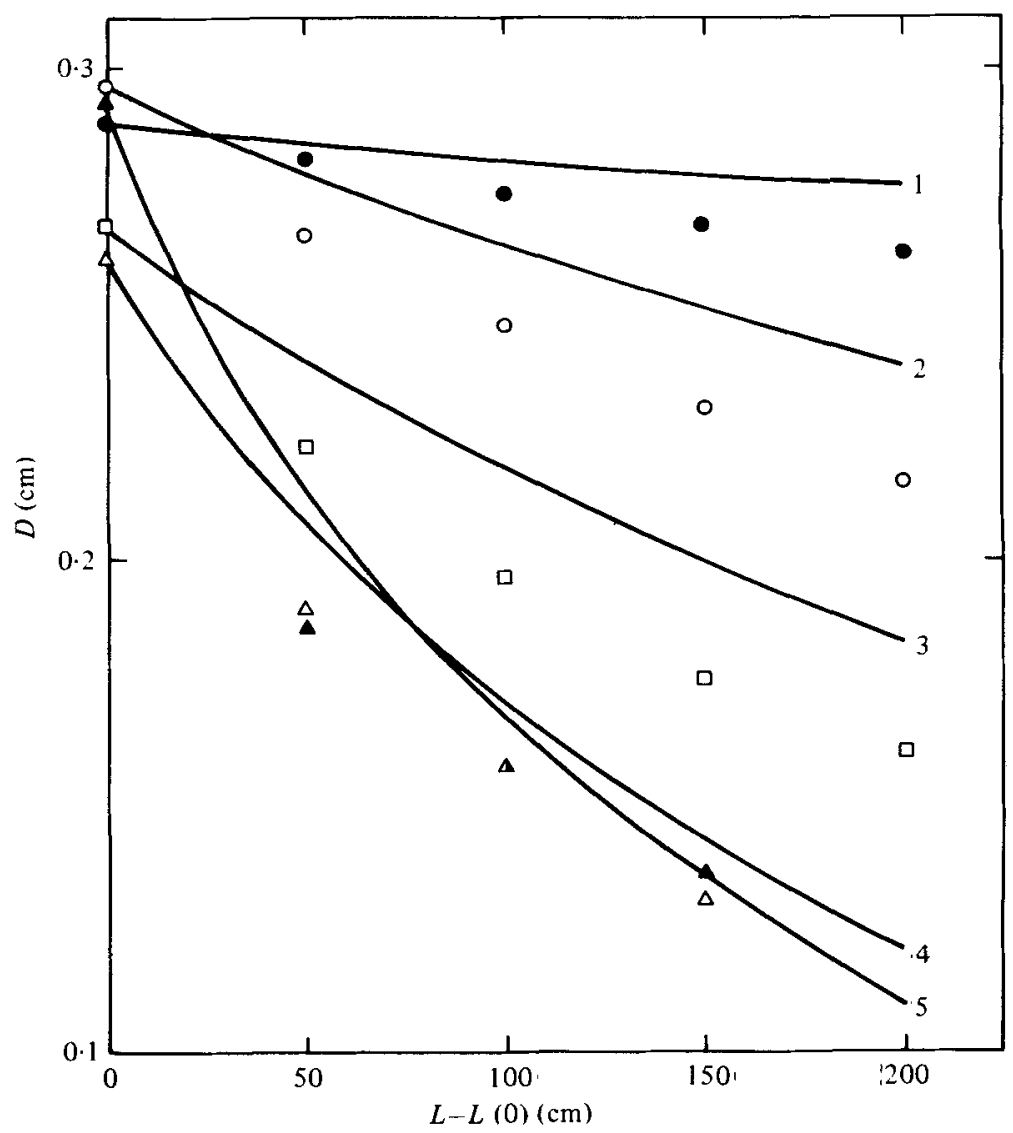

Figure 9. A comparison of experimental trajectory of a deformable drop in a three-dimensional Poiseuille flow with present theory (8.5) (-). From Goldsmith \& Mason (1962), $\kappa=2 \times 10^{-4}$, $\mu_{0}=50 \mathrm{P}, B_{0}=0.4 \mathrm{~cm}, \sigma=29 \mathrm{dyne}_{\mathrm{cm}}^{-1}$. $O$, case $1: a=0.0175 \mathrm{~cm}, V_{\max }^{*}=0.142 \mathrm{~cm} \mathrm{~s}^{-1}$; $O$, case $2: a=0.0300 \mathrm{~cm}, V_{\max }^{*}=0.142 \mathrm{~cm} \mathrm{~s}^{-1} ; \square$, case $3: a=0.0390 \mathrm{~cm}, V_{\max }^{*}=0.142 \mathrm{~cm}$ $\mathrm{s}^{-1} ; \triangle$, case $4: a=0.0410 \mathrm{~cm}, V_{\max }^{*}=0.283 \mathrm{~cm} \mathrm{~s}^{-1} ; \Delta$, case $5: a=0.0350 \mathrm{~cm}, V_{\max }^{*}=0.565$ $\mathrm{cm} \mathrm{s}^{-1}$.

Goldsmith \& Mason (1962). From figure 9, we see that the agreement is good, although in all cases the observed rate of migration is slightly greater than the prediction. We believe that this discrepancy is due to wall effects which are neglected here and will tend to increase the rate. In support of this opinion is the fact that the error is largest for cases 1 and 2 in which the drop remains close to the boundary for a long time, but becomes smaller for cases 4 and 5 in which the drop migrates rapidly to the tube centre. In addition, Wohl \& Rubinow (1974) have also compared their migration velocity with the experimental observations, and claimed that their agreement was good. However, from their figure 9 , we see that the prediction is significantly greater than the measured rates in cases 3,4 and 5 . Furthermore, they have made an algebraic error, involving a factor of 2 , in their calculations. (When calculating the centre-line velocity, they used $V_{\max }^{*}=Q / \pi B_{0}^{2}$. The correct expression is $V_{\max }^{*}=2 Q / \pi B_{0}^{2}$.) If this factor is introduced into the calculations, their theory will over-estimate the migration rate by an even wider margin. 


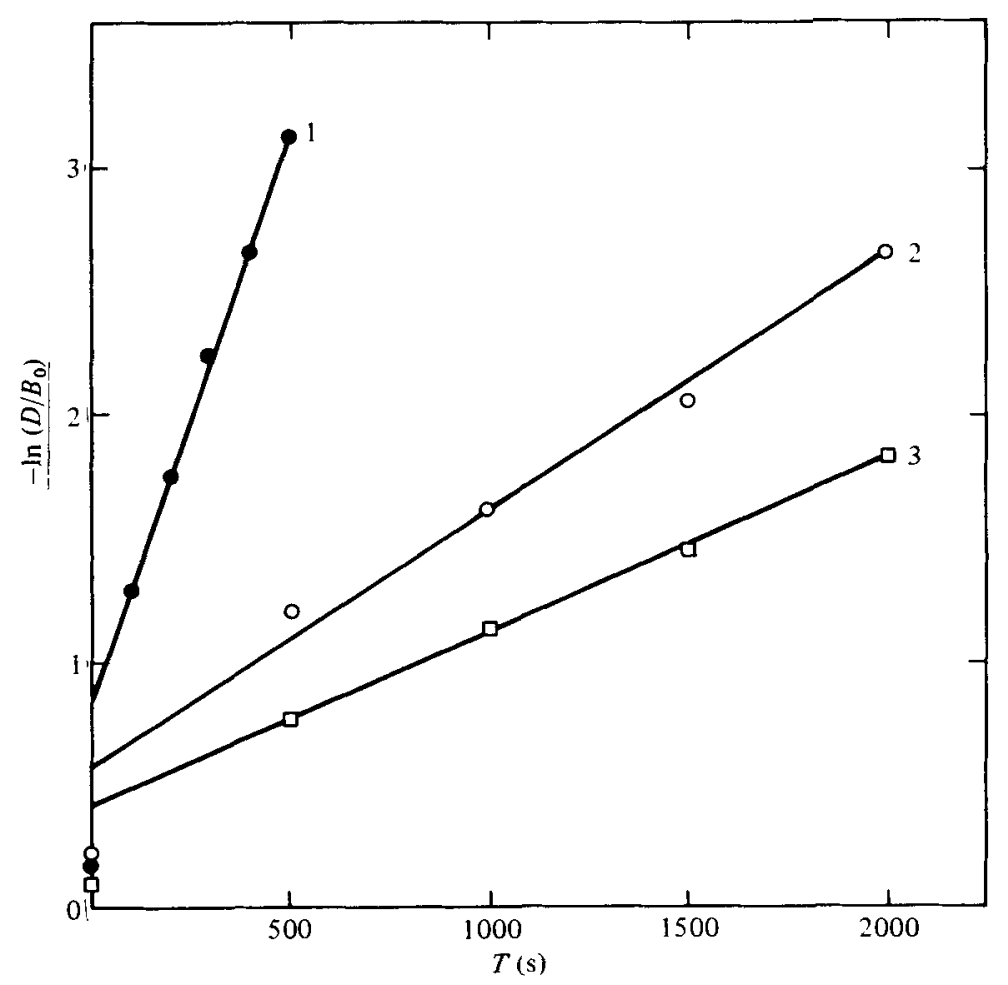

Fiqure 10. $-\ln \left(D / B_{0}\right)$ vs. $T$ for a deformable drop in a three-dimensional Poiseuille flow $\left(B_{0}=0.4 \mathrm{~cm}\right)$ of $3 \%$ PIB solution in Decalin, observed by Gauthier et al. $(1971 b)$. Case 1: $a=0.044 \mathrm{~cm}, V_{\max }^{*}=0.253 \mathrm{~cm} \mathrm{~s}^{-1} ; \bigcirc$, case $2: a=0.044 \mathrm{~cm}, V_{\max }^{*}=0.130 \mathrm{~cm} \mathrm{~s}^{-1} ; \square$, case $3: a=0.021 \mathrm{~cm}, V_{\max }^{*}=0.253 \mathrm{~cm} \mathrm{~s}^{-1} ; \ldots$, best linear fit with data.

Finally, we consider the problem of a Newtonian deformable drop in a nonNewtonian fluid. In a Couette flow, Gauthier et al. (1971 a) showed that a drop suspended in a $1.5 \%$ solution of PAA in water migrates to an equilibrium position between the centre-line and the outer cylinder. Here, there will be separate independent contributions from deformation and non-Newtonian effects. It is obvious that deformation alone results in a lateral force toward the centre-line. Normal stresses, on the other hand, will cause migration toward the outer cylinder, if we assume as before that the contribution from the shear gradient in a Couette device dominates over that from the particle interaction with the walls, particularly for a strong viscoelastic suspending medium which is not well modelled as a second-order fluid. On this basis, our theory indeed predicts an intermediate equilibrium position where the deformation and viscoelastic effects cancel each other. However, owing to the speculative nature of the above ideas, we shall not attempt a detailed comparison of theory with experimental data here.

For a three-dimensional Poiseuille flow, our theory may be compared in detail with the data of Gauthier et al. (1971b) for a $3 \%$ solution of PIB in Decalin. By integrating $\lambda\left(\hat{U}_{s}^{(\lambda)}\right)_{3}+\delta\left(\hat{U}_{s}^{(\delta)}\right)_{3}$, we obtain (for $\kappa=0$ )

$$
-\ln \frac{D}{B_{0}}+\ln \frac{D(0)}{B_{0}}=\left\{\left[-\frac{\phi_{3}}{\mu_{0}}\left(19+40 \epsilon_{1}\right)\right]\left[\frac{4 V_{\max }^{* 2} a^{2}}{63 B_{0}^{4}}\right]+\frac{16 V_{\max }^{* 2} a^{3} \mu_{0}}{7 B_{0}^{4} \sigma}\right\} T .
$$


In figure 10, we plot $-\ln \left(D / B_{0}\right)$ versus $T$ for three different cases. After subtracting the independent deformation contributions (i.e. $16 V_{\max }^{* 2} a^{3} \mu_{0} / 7 B_{0}^{4} \sigma$ ) from the slopes, we obtain numerical values for the parameter $-\phi_{3}\left(19+40 \epsilon_{1}\right) / \mu_{0}$ which are $5 \cdot 4,3 \cdot 2$ and $5 \cdot 4 \mathrm{~s}$ respectively. These values are apparently inconsistent with those obtained previously for a rigid sphere in a $6 \%$ solution of PIB in Decalin, since in general $\phi_{3} / \mu_{0}$ should decrease for decreasing concentrations of PIB (ef. Brodnyan, Gaskins \& Philippoff 1957). However, it must be noted that our present procedure for calculating $\phi_{3} / \mu_{0}$ is incomplete, since the effect of the interaction of a deformed drop with the bounding walls has not been accounted for [recall that even (8.6) shows a slight discrepancy with the experiments]. In the present case, since large drops are used, this contribution is likely to be large and may even be of the same order of magnitude as the normal stress term which we obtain above. To put it in another way, we hypothesize that the contribution to migration which we have so far attributed to normal stresses may yet come from wall effects in drop deformation.

This work was supported by a grant from the National Science Foundation.

\section{Appendix}

We shall consider briefly the theoretical basis for generalization of the theory for motion of a deformable drop from a two-dimensional to general quadratic flow of a Newtonian fluid. The equations of motion are given in (4.2) and (6.1), with boundary conditions from $(4.10 a)-(4.10 c)$ and $(6.3 a)-(6.3 c)$. Following the same procedures as Chan \& Leal (1977), we express the undisturbed velocity in index notation as

$$
\begin{aligned}
V_{i}= & \alpha_{i}+e_{i j} x_{j}-\epsilon_{i j k} \Omega_{k} x_{j}+\psi_{i j k} x_{k} x_{j}-\frac{1}{3}\left(\epsilon_{i j l} \delta_{m k}+\frac{1}{2} \delta_{i m} \epsilon_{l j k}\right) \theta_{l m} x_{k} x_{j} \\
& +\frac{1}{10}\left(-\delta_{k l} \delta_{i j}-\delta_{j l} \delta_{i k}+4 \delta_{i l} \delta_{j k}\right) \tau_{l} x_{k} x_{j}-\left(\hat{U}_{s}\right)_{i} .
\end{aligned}
$$

At $O(1)$, the translational velocity is

$$
\left(\hat{U}_{s}^{(0)}\right)_{i}=\alpha_{i}+\kappa \tau_{j} /(2+3 \kappa) .
$$

An expression for the deformation of the drop (cf. also Haber \& Hetsroni 1971) may be obtained at this stage by general tensorial arguments. In principle, it should include all linear terms from the six flow parameters. For our purpose, it is not necessary to obtain this expression in detail. However, we must note that eventually only $e_{i j}$ and $\psi_{i j k}$ will remain, since all other terms vanish in $(4.10 \mathrm{c})$.

By considering the force on the drop, the $O(\delta)$ migration velocity may also be obtained. Owing to the boundary conditions, it will in general include all terms that are quadratic in the flow parameters. Since the problem is spherically isotropic, we easily obtain

$$
\begin{aligned}
\left(\hat{U}_{s}^{(\delta)}\right)_{i}= & \frac{1+\kappa}{2 \pi(2+3 \kappa)}\left\{\hat{c}_{1}^{(1)}\left[\alpha_{m}-\left(\hat{U}_{s}^{(0)}\right)_{m}\right] e_{m i}+\hat{c}_{\mathbf{2}}^{(1)} \epsilon_{i m n}\left[\alpha_{m}-\left(\hat{U}_{s}^{(0)}\right)_{m}\right] \Omega_{n}\right. \\
& \left.+\hat{c}_{3}^{(1)} e_{n m} \psi_{m n i}+\hat{c}_{4}^{(1)} \epsilon_{i m n} e_{m l} \theta_{l n}+\hat{c}_{5}^{(1)} e_{i m} \tau_{m}+\hat{c}_{6}^{(1)} \Omega_{m} \theta_{m i}+\hat{c}_{7}^{(1)} \epsilon_{i m n} \Omega_{n} \tau_{m}\right\}
\end{aligned}
$$

By comparison with (6.6), we see that $\Omega_{i}$ cannot contribute to migration and hence $\hat{c}_{2}^{(1)}, \hat{c}_{6}^{(1)}$ and $\hat{c}_{7}^{(1)}$ are all zero. Combining $\hat{c}_{2}^{(1)}$ and $\hat{c}_{5}^{(1)}$, we finally simplify (A 3$)$ to

$$
\left(\hat{O}_{s}^{(\delta)}\right)_{i}=\frac{1+\kappa}{2 \pi(2+3 \kappa)}\left\{\hat{c}_{3}^{(1)} e_{n m} \psi_{m n i}+\hat{c}_{4}^{(1)} \epsilon_{i m n} e_{m l} \theta_{l n}+\hat{c}_{8}^{(1)} e_{i m} \tau_{m}\right\}
$$


The coefficients are obtained by a detailed comparison with our two-dimensional quadratic shear flow calculations, which then gives

and

$$
\left.\begin{array}{l}
\hat{c}_{3}^{(1)}=\frac{\pi}{14(1+\kappa)}\left[\frac{16+19 \kappa}{1+\kappa}(6+7 \kappa)-\frac{10+11 \kappa}{1+\kappa}\left(\frac{8-\kappa+3 \kappa^{2}}{1+\kappa}\right)\right], \\
\hat{c}_{4}^{(1)}=-\frac{\pi}{6(1+\kappa)}\left[\frac{16+19 \kappa}{1+\kappa}\left(\frac{2+2 \kappa+\kappa^{2}}{4+\kappa}\right)\right] \\
\hat{c}_{8}^{(1)}=-\frac{\pi}{10(1+\kappa)}\left[\frac{16+19 \kappa}{1+\kappa}\left(\frac{6-4 \kappa+3 \kappa^{2}}{2+3 \kappa}\right)\right] .
\end{array}\right\}
$$

For a Poiseaille flow in a pipe

and

$$
\left.\begin{array}{rl}
e_{n m} \psi_{m n i} & =\frac{1}{5} \beta \gamma \delta_{3 i} \\
\epsilon_{i m n} e_{m l} \theta_{l n} & =0 \\
e_{i m} \tau_{m} & =\beta \gamma \delta_{3 i} .
\end{array}\right\}
$$

Thus, (6.10) is finally obtained by substitution of (A 5) and (A 6) into (A 4).

\section{REFERENCES}

Beavers, G. S. \& JosePh, D. D. 1975 J. Fluid Mech. 69, 475.

Bretherton, F. P. 1962 J. Fluid Mech. 14, 284.

Brodnyan, J. G., Gaskins, F. H. \& Philippoff, W. 1957 Trans. Soc. Rheol. $1,109$.

Brunn, P. 1976 Rheol. Acta 15, 589.

Chaffey, C. E., Brenner, H. \& Mason, S. G. 1965 Rheol. Acta 4, 64.

Chaffey, C. E., Brenner, H. \& Mason, S. G. 1967 Rheol. Acta 6, 100.

Chan, P. C.-H. 1979 Ph.D. thesis, California Institute of Technology.

Chan, P. C.-H. \& Leal, L. G. 1977 J. Fluid Mech. 82, 549.

Cox, R. G. \& Brenner, H. 1968 Chem. Engng Sci. 23, 147.

Frankel, N. A. \& Acrivos, A. 1970 J. Fluid Mech. 44, 65.

Gadthier, F., Goldsmith, H. L. \& Mason, S. G. 1971 a Rheol. Acta 10, 344.

Gauthier, F., Goldsmith, H. L. \& Mason, S. G. 1971 b Trans. Soc. Rheol. 15, 297.

Goldsmith, H. L. \& Mason, S. G. 1962 J. Colloid Sci. 17, 448.

HABer, S. \& Hetsroni, G. 1971 J. Fluid Mech. 49, 257.

Hadamard, J. 1911 C. R. Acad. Sci. Paris 152, 1735.

Happei, J. \& Brenner, H. 1973 Low Reynolds Number Hydrodynamics. Noordhoff.

Ho, B. P. \& LEAL, L. G. 1974 J. Fluid Mech. 65, 365.

Ho, B, P. \& LeaL, L. G. 1976 J. Fluid Mech. 76, 783.

Karnis, A. \& Mason, S. G. 1966 Trans. Soc. Rheol. 10, 571.

Karnis, A. \& Mason, S. G. 1967 J. Colloid Sci. 24, 164.

LeAL, L. G. 1975 J. Fluid Mech. 69, 305.

Peery, J. H. 1966 Ph.D. thesis, Princeton University.

RybczYnski, W. 1911 Bull. Akad. Sci. de Cracovie, A 40.

TAYLOR, G. I. 1932 Proc. Roy. Soc. (London) A 138, 41.

TAylor, G. I. 1934 Proc. Roy. Soc. (London) A 146, 501.

WoHL, P. R. 1976 A.dv. Engng Sci., N.A.S.A. CP-2001. 4, 1493.

WoңL, P. R. \& Rubinow, S. I. 1974 J. Fluid Mech.62, 185. 